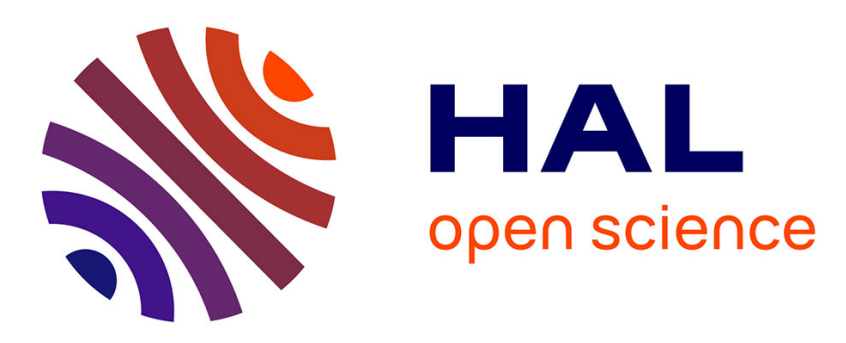

\title{
Le tri des déchets ménagers: une caractérisation des logistiques déployées par les consommateurs
}

\author{
Elisa Monnot, Fanny Reniou, Aurélien Rouquet
}

\section{To cite this version:}

Elisa Monnot, Fanny Reniou, Aurélien Rouquet. Le tri des déchets ménagers: une caractérisation des logistiques déployées par les consommateurs. Recherche et Applications en Marketing (French Edition), 2014, 29 (3), pp.74-98. 10.1177/0767370114527674 . hal-01123407

\section{HAL Id: hal-01123407 \\ https://hal.science/hal-01123407}

Submitted on 4 Mar 2015

HAL is a multi-disciplinary open access archive for the deposit and dissemination of scientific research documents, whether they are published or not. The documents may come from teaching and research institutions in France or abroad, or from public or private research centers.
L'archive ouverte pluridisciplinaire HAL, est destinée au dépôt et à la diffusion de documents scientifiques de niveau recherche, publiés ou non, émanant des établissements d'enseignement et de recherche français ou étrangers, des laboratoires publics ou privés. 


\title{
Le tri des déchets ménagers : une caractérisation des logistiques déployées par les
} consommateurs

\author{
Elisa MONNOT \\ Maître de Conférences \\ Université de Cergy-Pontoise \\ Thema, CNRS UMR 8184 \\ 33 boulevard du Port \\ 95011 Cergy Pontoise Cedex, France \\ Tél. : 0134252305 \\ elisa.monnot@u-cergy.fr \\ Fanny RENIOU \\ Maître de Conférences \\ Université Paris-Est Créteil \\ Institut de Recherche en Gestion \\ Place de la Porte des Champs, 4 route de Choisy \\ 94010 Créteil Cedex \\ Tél. : 0145171684 \\ fanny.reniou@u-pec.fr
}

\section{Aurélien ROUQUET}

Professeur de logistique

NEOMA Business School

Centre de Recherche sur le Management des Clients et CRET-LOG (Aix-Marseille-

\author{
Université) \\ 59, rue Pierre Taittinger \\ 51100 Reims, France \\ Tél. : 0326775746 \\ aurelien.rouquet@neoma-bs.fr
}

Cet article s'inscrit dans le cadre du programme de recherche Movida du Ministère en charge du Développement Durable. Les auteurs ont contribué à parts égales à l'élaboration de cet article. Les noms sont donc classés par ordre alphabétique. 


\title{
Le tri des déchets ménagers : une caractérisation des logistiques déployées par les consommateurs
}

\section{Résumé}

Cette recherche caractérise les logistiques déployées par les consommateurs pour trier leurs déchets ménagers. A travers l'analyse de vingt cas de trieurs, trois types de logistiques sont identifiés en fonction des caractéristiques du contexte de tri et du rapport souhaité aux déchets : la massification, le juste-à-temps et la mutualisation. Contribuant au courant de la consumer logistics, notre recherche montre que le tri n'est pas pour le consommateur qu'une question de motivation et de contexte mais également de logistique.

Mots clés : tri, logistique de consommation, déchets ménagers, supply chain, développement durable.

\section{Waste recycling: a characterization of logistics used by consumers}

\begin{abstract}
This research characterizes the logistics consumers use to recycle their household waste. Through the analysis of twenty cases of waste-recyclers, it identifies three types of logistics based on the characteristics of the recycling context and the desired relation to waste: massification, just-in-time and pooling. This research contributes to the Consumer Logistics research field, showing that recycling is not only a question of motivation and context, but also of logistics.
\end{abstract}

Keywords: recycling, consumer logistics, household waste, supply chain, sustainable development. 


\section{INTRODUCTION}

Revers de la société de consommation, les déchets constituent un enjeu crucial en termes de développement durable. Ceci est particulièrement vrai pour les déchets ménagers, c'est-à-dire les «déchets provenant de l'activité domestique des ménages et dont l'élimination relève généralement de la compétence des communes ${ }^{1} \gg($ ADEME, 2012). Leur production annuelle par habitant a doublé en cinquante ans. Pour mettre en place un développement durable, un «point de passage obligé » (Callon, 1986) est ainsi d'améliorer la participation des consommateurs au recyclage des déchets, par le biais du tri (Rumpala, 1999; Savaskan, Bhattacharya et van Wassenhove, 2004). Or, les contributions des consommateurs au tri sont très disparates.

Depuis des décennies, plusieurs travaux ont tenté d'expliquer cette disparité des niveaux de tri. Ils se sont intéressés à ses déterminants sociodémographiques - âge, revenu, niveau d'éducation, sexe - à ses motivations, liées à des facteurs internes, d'une part - attitude environnementale, croyances, valeurs - et externes, d'autre part - normes sociales, incitations financières, lois (Granzin et Olsen, 1991 ; Shrum, Lowrey et McCarty, 1994 ; Hornik et Cherian, 1995 ; Biswas et alii, 2000 ; Kreziak et Cova, 2010 ; Fiorello, 2011). D'autres travaux ont souligné le rôle des déterminants contextuels liés au «lieu» de tri, comme la place au domicile ou la distance des points de collecte (Humphrey et alii, 1977 ; De Young, 1988/1989 ; Vining et Ebreo, 1990 ; Oskamp et alii, 1991 ; Heckler, 1994 ; Jolivet, 1999 ; Desjeux, 2002). Toutefois, ces recherches ne suffisent pas à expliquer la disparité des niveaux de tri et appellent au développement d'approches alternatives.

Cette recherche suggère qu'un aspect complémentaire doit être pris en compte en vue de comprendre le comportement de tri : l'aspect «logistique» du tri. Quels que soient ses motivations et le contexte dans lequel il agit, le consommateur qui veut trier doit en effet être capable de surmonter de nombreux problèmes «logistiques » (Granzin et Bahn, 1989). Quels types de bacs utiliser? Où stocker les déchets? Quand et comment les expédier ? Etc. Pour le consommateur, l'effort est d'autant plus grand qu'il ne s'agit pas simplement de résoudre un à un ces problèmes mais de coordonner les trois activités de la logistique du tri : séparation des déchets, stockage et expédition (Pieters, 1991), et ce dans un contexte qui n'est pas toujours favorable (Thøgersen, 1997 ; Bekin, Carrigan et Szmigin, 2007). Ainsi, s'intéresser à la mise en place de cette logistique pourrait apporter un éclairage à la littérature sur le tri.

1 Cette définition de l'Agence De l'Environnement et de la Maîtrise de l'Energie (ADEME) inclut les ordures ménagères ainsi que les déchets encombrants et dangereux. Dans cette recherche, nous excluons ces déchets encombrants et dangereux qui relèvent d'autres logiques. 
D’un point de vue théorique, cette logistique déployée par les trieurs est l'une des multiples logistiques que les individus mettent en place dans leur consommation et qui sont au cœur d'un courant de recherche en marketing, celui de la consumer logistics (Granzin et Bahn, 1989 ; Granzin, Painter et Bahn, 2005 ; Teller, Kotzab et Grant, 2012). Bien que l'aspect logistique soit qualifié par ce courant de déterminant pour comprendre la consommation, il a été peu approfondi dans la littérature en marketing en général, et sur le tri en particulier. Dans ce cadre, l'objectif de cet article est de caractériser cette pratique post-consommation qu'est la logistique du tri. Précisément, notre question de recherche est la suivante: comment les consommateurs assurent-ils et coordonnent-ils la logistique requise par le tri des déchets ménagers? La thèse défendue dans cet article est ainsi que le tri est non seulement une question de motivation et de contexte mais également de logistique.

Pour répondre à cet objectif, l'article passe d'abord en revue la littérature sur les déterminants individuels et contextuels du niveau de tri et met en évidence l'intérêt de caractériser les logistiques déployées par les consommateurs pour trier. Il décrit ensuite la méthodologie qui s'appuie sur l'étude de vingt cas de logistiques du tri mises en place par des trieurs. Une partie consacrée aux résultats montre que les consommateurs déploient trois types de logistiques pour trier - la massification, le juste-à-temps et la mutualisation - en fonction : (1) des caractéristiques du contexte dans lequel ils se trouvent et (2) du rapport souhaité aux déchets. Pour mettre en avant sa contribution, cet article discute les apports théoriques de la recherche à la littérature en marketing sur le comportement de tri ainsi qu'au courant de la consumer logistics. La discussion souligne, d'une part, que pour mieux comprendre le tri, il est nécessaire de dépasser l'analyse des déterminants du niveau de tri et de s'intéresser aux logistiques des consommateurs. Elle analyse, d'autre part, les similarités et différences entre entreprises et consommateurs en matière de gestion logistique des flux. Enfin, cet article propose des pistes managériales pour développer les capacités logistiques des consommateurs et identifie les perspectives de recherche méritant d'être poursuivies.

\section{REVUE DE LITTERATURE}

Si la majorité des recherches sur le tri s'intéresse aux déterminants individuels et contextuels du niveau de tri, d'autres analysent le processus de tri, comprenant plusieurs activités et pouvant requérir, selon le contexte, plus ou moins d'efforts. Le fait qu'il soit lié à des activités de manutention, de stockage, d'expédition et qu'il implique un objectif, un espace, des moyens et de l'énergie témoigne de toute la logistique nécessaire au tri. Le courant de la 
consumer logistics (Granzin et Bahn, 1989), qui s’intéresse aux logistiques inhérentes à la consommation, peut dès lors apporter un éclairage intéressant dans le cas du tri.

\section{Un état des recherches sur les déterminants individuels et contextuels du tri}

Depuis les années 1970, de nombreux travaux en marketing ont été conduits sur le recyclage et en particulier sur le comportement de tri (Kreziak et Cova, 2010). Ils ont principalement exploré l'influence sur ce dernier de déterminants sociodémographiques - âge, revenu, niveau d'éducation, sexe - ainsi que des motivations, liées à des facteurs internes - attitude environnementale, croyances, valeurs - et externes - normes sociales, incitations financières, lois (Granzin et Olsen, 1991 ; Shrum, Lowrey et McCarty, 1994 ; Hornik et Cherian, 1995 ; Biswas et alii, 2000 ; Kreziak et Cova, 2010 ; Fiorello, 2011). Toutefois, ces recherches, qui réduisent le comportement de tri à un niveau de tri ${ }^{2}$, divergent souvent dans leurs résultats (Annexe A1). D'autres travaux, dont les résultats sont plus convergents, soulignent la forte influence des déterminants contextuels, c'est-à-dire du lieu au sein duquel les individus trient. Ils évoquent, d'une part, l'influence des caractéristiques des domiciles, en particulier de la place disponible, sur le niveau de tri (Berger, 1997 ; Jolivet, 1999). Ainsi, selon Desjeux (2002), la mise en œuvre d'une politique de tri des déchets peut paraître aller de soi mais s'il n'existe pas d'espace suffisant pour mettre plusieurs poubelles dans la cuisine, cette politique risque d'échouer en raison de la contrainte de place. D' autre part, ils soulignent l'influence du système de collecte mis en place dans la commune et notamment de la localisation et la distance des points de collecte, la disponibilité et la commodité d'usage des conteneurs, ou la qualité de service du ramassage (Humphrey et alii, 1977 ; De Young, 1988/1989 ; Vining et Ebreo, 1990 ; Oskamp et alii, 1991 ; Pieters, 1991 ; Heckler, 1994 ; Berger, 1997). Thøgersen (1997) note que la commodité renvoie à la fois à la présence ou l'absence de conteneurs de tri et à des éléments variables selon les ménages, comme par exemple la distance aux points de collecte. Ainsi, plus les individus sont éloignés des conteneurs, moins ils ont tendance à trier. Les modalités définies par les collectivités conditionnent le niveau de tri des consommateurs en influençant leur perception des contraintes liées à cette activité (Jolivet, 1999). Tous ces facteurs sont autant de barrières à l'adoption du tri (Boldero, 1995 ; Davies, Foxall et Pallister, 2002) ou de contraintes contextuelles que Dahab, Gentry et $\mathrm{Su}$ (1995) conceptualisent sous le terme d'efforts perçus. Prises ensemble, ces recherches sur les déterminants du tri ne suffisent toutefois pas à expliquer les disparités de niveaux de tri.

\footnotetext{
${ }^{2}$ Ce niveau est évalué à travers le type de déchet trié (journaux, bouteilles) et la fréquence de tri, mesurée à l'aide d'échelles de Likert allant de « toujours » à « jamais » ou de «très fréquemment » à « rarement » (Jackson et alii, 1993 ; Shrum, Lowrey et McCarty, 1994 ; Biswas et alii, 2000 ; McCarty et Shrum, 2001). Ces mesures sont très hétérogènes (Shrum, Lowrey et McCarty, 1994) et s'appuient sur du déclaratif.
} 
Au-delà de ses motivations et du contexte dans lequel il agit, le consommateur doit, pour faire le tri, résoudre des problèmes « logistiques » qu'il conviendrait d'analyser pour parvenir à une meilleure compréhension du comportement de tri (Granzin et Bahn, 1989). Les quelques recherches s'étant intéressées à cet aspect logistique soulignent que le comportement de tri peut être scindé en trois activités logistiques de séparation, stockage et expédition (Pieters, 1991). Il faut d'abord pour le consommateur disposer de réceptacles particuliers (sacs, boîtes ou poubelles à compartiments) afin de séparer les déchets (en fonction du matériau, des composants ou des types de produits). Il faut ensuite déterminer où les déchets doivent être stockés au domicile. Il faut enfin gérer l'expédition des déchets, qui implique un déplacement plus ou moins long en fonction de la localisation des points de collecte. Ces recherches, essentiellement descriptives, indiquent que ces activités peuvent avoir un coût pour le consommateur qui peut déterminer son comportement de tri (Pieters, 1991, p. 66 ; Bekin, Carrigan et Szmigin, 2007) mais elles ne permettent pas de comprendre comment les consommateurs réalisent et coordonnent la logistique du tri, en fonction de leur contexte.

Cette question des logistiques inhérentes aux actes de consommation est au cœur d'un courant de recherche en marketing, celui de la consumer logistics (Granzin et Bahn, 1989; Granzin, Painter et Bahn, 2005), qui envisage la participation des consommateurs à la prise en main des produits depuis le point d'acquisition jusqu'au point de consommation ou de destruction. Ce courant propose d'analyser les activités logistiques des individus durant la consommation à l'aide des travaux menés sur la logistique des entreprises. En effet, l'enjeu pour les consommateurs est bien similaire à celui des entreprises. Dans les deux cas, il s'agit d'assurer et de coordonner des activités physiques (Heskett, 1977): de l'approvisionnement au transport, en passant par la production et le stockage, qui comprend la constitution de lots (kitting), leur préparation (picking) et leur emballage (packing). Cette coordination s'inscrit dans le cadre d'une chaîne logistique (supply chain), définie comme l'ensemble des infrastructures et processus nécessaires pour fournir des produits/services, d'un point de départ à un destinataire final (Christopher, 2005). L'objectif est de piloter les flux physiques (Tixier, Mathe et Colin, 1983) en tenant compte à la fois des aspects internes (l'entreprise) et externes (les partenaires) de la supply chain. Dès lors, pour mieux comprendre et caractériser les logistiques inhérentes aux activités de consommation, Granzin et Bahn (1989) proposent de transférer au consommateur les cinq fonctions du «système logistique » des entreprises (Bowersox, 1978) : localisation, transport, inventaire, stockage et information. Ils soulignent toutefois que des spécificités existent au niveau du consommateur. Notamment, la localisation 
du domicile est plus essentielle pour le consommateur car elle structure l'organisation des logistiques de la consommation. Plus fondamentalement, Granzin et Bahn (1989) appellent à utiliser l'éclairage d'autres disciplines des sciences sociales, comme le comportement du consommateur, la sociologie, la psychologie ou l'anthropologie pour saisir la spécificité de la logistique de consommation (Rychlak, 1977; Werner, 1987). Contribution séminale et fondamentale, la recherche de Granzin et Bahn (1989) n'a cependant été que peu approfondie. Ainsi, l'approche de ces auteurs reste descriptive et ils ne caractérisent pas les logistiques déployées par les individus, ce qu'ils considèrent d'ailleurs eux-mêmes comme une limite. De plus, les travaux en consumer logistics se sont jusqu'à présent focalisés sur une étape de la consommation : le shopping. Granzin, Painter et Valentin (1997) ont notamment identifié six segments de shoppers en fonction de leur type de participation à la logistique et de leurs caractéristiques sociodémographiques et comportements d'achat. Plus récemment, Teller, Kotzab et Grant (2012) montrent que les activités logistiques peuvent être un moyen de comprendre le choix d'un format de distribution par un client : la logistique qu'il doit prendre en charge a en effet un impact sur la commodité du shopping. Enfin, Goudarzi et Rouquet (2013) ont récemment mis en évidence le fait que les consommateurs pouvaient jouer quatre rôles logistiques dans la distribution. Dès lors, l'objectif de cette recherche, qui s'inscrit dans le courant de la consumer logistics, est de caractériser les logistiques déployées par les consommateurs au cours d'une étape de la consommation : le tri.

\section{METHODOLOGIE DE LA RECHERCHE}

L'objectif de la recherche conduit à étudier une activité complexe, celle de la logistique qui permet à un consommateur de trier. Dès lors, une approche qualitative est préférée car elle permet de restituer toute la complexité de l'activité. Comme notre objet de recherche, la logistique du tri, est un flux physique composé de plusieurs activités ou étapes, nous avons opté pour une recherche processuelle (Van de Ven, 1992 ; Pettigrew, 1997 ; Langley, 1999) qui permet d'étudier l'évolution d'une variable dans le temps (Grenier et Josserand, 1999).

\section{Collecte des données : une combinaison d'entretiens et d'observations avec photographies}

L'unité d'analyse retenue pour étudier la logistique du tri est l'individu dans un lieu. En effet, selon la littérature, le tri est influencé par le contexte de l'individu, c'est-à-dire par les caractéristiques de son domicile et le type de système de collecte proposé $e^{3}$. Il est dès lors

\footnotetext{
${ }^{3}$ Trois systèmes sont possibles : (1) un système de dépôt, les individus ne disposant au domicile que d'un seul conteneur pour tous les déchets ménagers ; (2) un système de collecte partiel, excluant le dépôt du verre, qui se fait alors dans des points de collecte extérieurs au domicile; (3) un système de collecte complet, tous les conteneurs de tri étant proposés au domicile (déchets organiques ; emballages cartonnés et plastiques ; verre).
} 
crucial de le prendre en compte, un même individu pouvant trier dans sa résidence principale et non dans sa résidence secondaire, ou inversement. Pour constituer l'échantillon, nous avons donc porté une attention particulière au contexte dans lequel le tri était réalisé, tant d'un point de vue interne (type de domicile: appartement ou maison; taille du domicile: T1 à T5) qu'externe (type de système de collecte : dépôt, partiel ou complet; type de zone : rurale ou urbaine ; département). Conformément au vocabulaire de la logistique, nous utilisons dans la suite de l'article le terme de «supply chain» interne/externe pour faire référence à ce contexte. Notre échantillon comporte ainsi vingt cas d'individus trieurs dans des lieux différents. Bien qu'elles ne soient pas centrales, les variables d'âge, de profession et de nombre de personnes dans le foyer ont été relevées a posteriori pour caractériser l'échantillon (Annexe A2). Les cas ont donc été choisis compte tenu de leur caractère dissemblable (Miles et Huberman, 2003) pour faciliter la comparaison inter-cas (Lincoln et Guba, 1985).

Tout d'abord, un entretien était réalisé au domicile auprès de la personne se déclarant être la plus responsable du tri des déchets au sein du foyer. Il était mené à l'aide d'un guide d'entretien semi-directif, centré autour des trois activités de la logistique du tri : séparation, stockage et expédition (Pieters, 1991). Ceci est cohérent avec le fait que, pour conduire une recherche sur le processus, il faut décomposer la variable processuelle en sous-variables. Le but pour le répondant était d'expliciter la manière dont la logistique du tri était réalisée à son domicile; son discours se focalisait alors sur les activités logistiques gérées pour trier. Les entretiens, systématiquement enregistrés et retranscrits, constituent un corpus de 304 pages. Des observations étaient également réalisées par les chercheurs lors de la visite au domicile. Le recours à l'observation se justifiait par la forte évidence physique des activités logistiques. Les cuisines, espaces domestiques dans lesquels les pratiques quotidiennes, et donc les déchets, sont particulièrement présentes (Southerton, 2001), ont été le lieu privilégié des observations. Celles-ci se sont également déroulées dans les salles de bain, chambres, garages et locaux à poubelles. Pour guider les observations, une grille avait été formalisée pour relever les principaux points à observer : emplacement des poubelles dans les pièces ; type et taille ; objets utilisés pour trier, stocker et transporter les déchets ; astuces utilisées, etc. Cette grille était ouverte et alimentée au fil des observations. Les répondants proposaient fréquemment au chercheur de constater, sur place, ce qu'ils évoquaient. L'entretien continuait ainsi devant les lieux physiques du tri, les répondants explicitant leurs actions en contexte. Lorsqu'elle n'était pas initiée par le répondant lui-même, cette démarche était encouragée par les chercheurs. Le fait de combiner observations et entretiens permettait d'assurer une meilleure fiabilité des données et de favoriser l'élicitation des comportements : le répondant s'en souvenait mieux en 
montrant des éléments au chercheur et ce dernier évitait ainsi une mauvaise compréhension des points évoqués. De plus, les observations pouvaient être utilisées au cours de la visite pour insister sur des points importants qui n'étaient pas spontanément évoqués par le répondant. Des photographies de ces éléments ont été prises par les chercheurs au domicile et aux points de collecte. Ceci a permis de constituer un corpus de 264 photographies.

\section{Procédure d'analyse des données : une analyse de contenu thématique}

L'analyse des données s'est faite en respectant la démarche qui caractérise l'analyse de contenu thématique (Kolbe et Burnett, 1991; Miles et Huberman, 2003). L'équipe de chercheurs a tout d'abord réalisé un codage descriptif des données. L'objectif était d'identifier des catégories ou concepts de premier niveau en vue de décrire les vingt logistiques observées. Ce codage descriptif a favorisé le développement d'un codage de deuxième niveau qui a permis de regrouper et classer les catégories descriptives initialement mises en évidence en catégories supérieures. Enfin, ces catégories de deuxième niveau ont été agrégées dans des thèmes plus généraux que sont: (1) les activités logistiques du tri, en accord avec la proposition de Pieters (1991); (2) les caractéristiques de la supply chain interne et externe, telles qu'elles ont été présentées par les répondants eux-mêmes, et regroupant les codes liés au milieu-contexte au sein duquel le processus se déroule (Miles et Huberman, 2003) ; (3) le rapport aux déchets, qui regroupe les codes liés à l'interprétation par les acteurs du processus et; (4) les caractéristiques des trieurs, qui regroupent les codes relatifs aux acteurs du processus. De manière classique, ce processus de codage a été réalisé par l'équipe de chercheurs en procédant par allers et retours entre les données et le système de code (AllardPoesi, 2003 ; Miles et Huberman, 2003).

Afin d'assurer la validité de la recherche, des précautions ont été prises. Les chercheurs ont fait tout au long du processus de recherche des points réguliers. Pour veiller à l'harmonisation de la collecte des données, les premières logistiques du tri ont été étudiées simultanément par les chercheurs, ce qui a permis de clarifier la manière dont les questions devaient être posées aux répondants et d'identifier les types de lieux et objets à observer. La grille de codage a été élaborée conjointement puis testée pour coder plusieurs logistiques, ce qui a permis de s'accorder sur les modalités précises du codage. Le codage des logistiques a été réparti de façon à ce que chaque entretien soit codé par au moins deux chercheurs. Si des divergences apparaissaient, elles étaient systématiquement débattues pour arriver à un consensus. Les analyses ont donc fait l'objet d'une triangulation entre les chercheurs pour vérifier la reproductibilité et donc la fiabilité de la recherche. 


\section{RESULTATS}

L'articulation entre les différents thèmes issus de notre analyse (Annexe A3) a conduit à identifier trois types de logistiques déployées par les consommateurs pour trier leurs déchets : la massification, le juste-à-temps et la mutualisation. Nous caractérisons successivement ces trois logistiques et mettons en évidence le fait que chacune d'elles : se traduit par un processus distinct de tri en termes de séparation, stockage et expédition; se retrouve dans un type de supply chain différent, dont le caractère favorable, ou non, est évalué par l'individu lui-même ; induit un rapport spécifique aux déchets, tant sur le plan physique, spatial que psychique ; et est plus ou moins adaptée à certains individus.

Un premier type de logistique du tri : la «massification»

Massifier les flux en interne avant de les pousser vers l'externe

La première logistique du tri déployée par les individus est la massification. Elle consiste à dédier un espace important au stockage et à la séparation des déchets au domicile. Cet espace, le plus souvent isolé dans une arrière-cuisine, un garage, un sous-sol ou un jardin, a pour fonction de centraliser tous les flux de déchets générés dans les différentes pièces du domicile. Il comprend en général plusieurs bacs de tri dédiés au stockage d'un type de déchet. Ces bacs, généralement fournis par la mairie, sont souvent de forte capacité et conduisent l'individu à réaliser la séparation des déchets au domicile: «moi il se trouve que c'est une maison donc on a deux poubelles individuelles, des bacs, des conteneurs individuels qu'on a eus il y a bien 18 ans, un truc comme ça, par la ville de Paris, ça fait longtemps déjà qu'on fait le tri (...) dans la mesure où j'ai un sous-sol qui me sert d'espace de rangement et de tri »(Martine). Une telle logistique permet de stocker un grand volume de déchets et conduit à aller peu fréquemment jusqu'aux points de collecte. L'expédition des déchets se fait en général lorsque les stocks débordent et que plus rien ne peut être entreposé, ce qui entraîne la mise en place d'un flux massif de déchets vers l'extérieur : "le verre, on n'a pas tant que ça de verre qui s'accumule, et ça peut attendre un peu (...) oh pour le verre, ça peut attendre trois semaines, un mois (...) la plupart du temps j'attends vraiment qu'il y ait un gros cumul, un grand sac bien plein de plein de bouteilles pour aller les porter »(Célia).

\section{Utiliser la supply chain interne face aux contraintes de la supply chain externe}

Cette logistique est déployée lorsque la supply chain interne est considérée par l'individu comme favorable, en termes de stockage, et la supply chain externe défavorable, en termes d'expédition. Elle se retrouve typiquement dans les maisons éloignées des points de collecte, où il faut parfois faire des kilomètres pour aller déposer les déchets. Dans ce cas, pour éviter 
un trajet dédié, les individus profitent d'autres déplacements pour les expédier: " c'est suffisamment grand pour que j'aie une poubelle à l'intérieur de la cuisine, pour jeter rapidement les choses (...) j'ai une poubelle à l'extérieur, j'ai même deux poubelles dans lesquelles je jette tout ce qui est gros. Parce que je ne veux pas que la poubelle à l'intérieur de la cuisine se remplisse trop vite. C'est plutôt pour les petites choses (...) pour aller au verre, c'est au village (...) ça prend un peu plus de temps... C'est au moins dix minutes, un quart d'heure (...) c'est quand j'ai besoin d'aller à la Poste ou à la ville. Mais j'y vais rarement spécialement»(Célia).

Une mise à distance spatiale et psychique des déchets, mais une présence physique

Cette logistique du tri induit une certaine mise à distance spatiale des déchets (Pierre, 2002). Ceux-ci étant cantonnés dans un espace dédié et isolé, la proximité olfactive et visuelle s'en trouve réduite (Douglas, 2001). Cette logistique implique également une mise à distance psychique des déchets, les individus n'ayant pas besoin de s'en préoccuper quotidiennement. Une telle logistique induit toutefois une présence physique forte des déchets. D'une part, les individus doivent manipuler des stocks lourds et volumineux au moment de l'expédition. D'autre part, ils sont contraints d'effectuer des flux internes entre les diverses zones de stockage temporaire (petites poubelles transitoires) et l'espace dédié (bacs de tri), multipliant ainsi les gestes de dépotage/rempotage. Le cas suivant illustre le rapport aux déchets induit par la massification : "j'ai une femme de ménage qui fait ce travail, qui descend la poubelle de salle de bain, qui ramasse avec un sac tous les déchets des salles de bain, et qui complète la poubelle de cuisine... et puis descend son sac plastique dans la poubelle à couvercle jaune (...) Donc cette poubelle ne sort pas toutes les semaines... mais comme elle est au sous-sol, il y fait relativement frais, donc il n'y a pas de pourrissement, d'odeurs... »(Clément).

Une logistique inadaptée aux individus ayant des difficultés physiques

$\mathrm{Du}$ fait de la forte présence physique des déchets, qui sont ici stockés en masse dans des conteneurs volumineux et lourds, les individus qui rencontrent des difficultés physiques sont moins enclins à opter pour ce type de logistique. Ainsi par exemple, une vieille dame vivant seule dans une grande maison pourrait éviter la massification en raison des contraintes physiques qu'elle représente. Si l'âge ou le sexe peuvent expliquer des capacités physiques limitées, elles peuvent aussi et surtout être liées à un handicap ou des problèmes de santé : «il arrive que ce soit moi, en faisant des rangements ou en triant des catalogues, etc. si la poubelle est pleine et elle est lourde, je ne la laisse pas à la femme de ménage parce que je suis encore assez valide pour le faire moi-même »(Clément). 
Un deuxième type de logistique du tri : le «juste-à-temps »

Une expédition des déchets en flux tirés par la production

La seconde logistique du tri est le juste-à-temps. Elle consiste à expédier les déchets rapidement, dès qu'ils sont produits, au moyen de trajets spécifiques jusqu'aux points de collecte. Les individus entreposent le plus souvent les déchets à recycler à même le sol de leur cuisine, à côté d'une poubelle dédiée aux déchets non recyclables : « [je la sors] une fois tous les deux jours, en moyenne (...) Quand je rentre, si je vois que la poubelle est pleine, je la sors directement... si jamais elle se remplit pendant la soirée, à ce moment-là je la sors quand même le soir. J'attends pas le lendemain (...) Comme je ne génère pas beaucoup de déchets de bouteilles, je stocke dans ma cuisine, même pas dans un carton, je le mets simplement à côté de la poubelle...» (Simon). Ils utilisent parfois un coin de couloir, dans l'entrée de l'habitation, pour stocker les déchets avant leur expulsion, libérant ainsi l'espace de stockage principal. Pour préparer l'expédition, les déchets sont rassemblés selon une méthode pick then pack (Mocellin, 2011) : les individus prélèvent les déchets au domicile puis les mettent dans un contenant utilisé pour le transport, ce qui les conduit à réaliser la séparation devant les points de collecte: "n'ayant pas de place, je mélange mon plastique avec mon verre chez moi, et après je suis obligé, quand je descends pour jeter, de tout poser et de prendre, et de faire ma répartition en bas »(Boris).

Utiliser la supply chain externe face aux contraintes de la supply chain interne Cette logistique est déployée lorsque les individus estiment qu'il est relativement simple d'expédier les déchets hors du domicile, car les points de collecte sont physiquement proches, et lorsqu'ils se sentent contraints sur le plan interne. Comme l'espace de stockage est ici limité, des solutions inventives ou temporaires sont parfois trouvées, comme par exemple le stockage vertical, le gerbage de plusieurs contenants ou le détournement de déchets en réceptacles : «je superpose par-dessus cette poubelle un sac pour mettre mes autres déchets, comme les bouteilles en verre (...) ça m'arrive d'utiliser les boîtes de lait de ma fille ou des gros cartons d'emballage pour stocker d'autres déchets comme des papiers de bureau ou les petits cartons autour des yaourts »(Elodie). Les individus soulignent que si leur domicile, et en particulier sa taille, le permettait, ils procéderaient différemment, en séparant par exemple les déchets chez eux plutôt qu'aux points de collecte : «j'ai pas beaucoup de place, mon coin poubelle il est très réduit, et puis en même temps il est à proximité de la chambre. Donc bon, tout ça mis bout à bout, moi faut que ça parte vite (...) Si j’avais la place je le ferais, si j'avais une grande cuisine je le ferais, si j’étais en maison évidemment que je le ferais! Je prends l'exemple d'un couple d'amis, chez eux ils sont organisés comme ça, où ils ont déjà 
directement des bacs en fonction du tri »(Boris).

Une mise à distance physique des déchets, mais une présence spatiale et psychique Cette logistique induit une certaine mise à distance physique des déchets (Pierre, 2002). Le volume et le poids des déchets à manipuler et expulser, à chaque fois, sont peu importants du fait des expéditions régulières. Le fait de consacrer un trajet dédié à l'expulsion des déchets permet également de diminuer le contact physique avec des déchets ou des conteneurs souvent perçus comme sales (Douglas, 2001). L'individu a en effet la possibilité de revenir à son domicile pour se laver les mains : "la poubelle de tri, on peut pas l'ouvrir, elle est scellée, et t'as une espèce de petite fente devant avec des lamelles en plastique ou j'sais pas quoi, et on a toujours l'impression que c'est super dégueulasse et je dois mettre mes mains dedans. Ce qui fait que plein de fois, je sais que j'me dis que je vais pas descendre la poubelle de tri maintenant, en allant faire mes courses ou me balader parce qu'après j'aurai les mains sales. Donc... je descends [dans la cour de l'immeuble], je mets les mains dedans, c'est sale, mais au moins, après je reviens, je vais me laver les mains » (Elodie). Le problème d'une telle logistique est qu'elle induit une présence spatiale des déchets, le stockage temporaire s'opérant souvent dans des zones de vie et de passage au sein du domicile. Enfin, le fait d'avoir à expulser presque quotidiennement les déchets entraîne une préoccupation psychique pour les individus, qui ne doivent pas les oublier. Ceci est d'autant plus le cas si une seule personne du ménage est chargée de l'expédition. Celle-ci peut parfois ressentir une certaine lassitude du fait qu'elle est la seule à devoir penser aux déchets : "c'est plutôt un peu par rébellion, la poubelle normale, que je la mets là [dans l'entrée]. C'est parce que, justement, ça m'agace de le faire toute seule. J'aimerais bien que mon mari s'en occupe un peu plus. C'est pas un plaisir, c'est pas une corvée, mais c'est pas une tâche hyper épanouissante quand même non plus. Donc, j'aimerais bien que ce soit un truc qui soit un peu partagé. Et donc le fait de la mettre là, c'est aussi pour essayer d'attirer sa curiosité »(Elodie). Se retrouve ici la question de «l'inaccessible égalité ménagère » (Kaufmann, 1993).

Une logistique inadaptée aux individus ayant besoin d'ordre, tendance à tout garder ou à procrastiner

Cette logistique, synonyme de présence spatiale des déchets, peut poser problème aux individus qui ont un besoin d'ordre élevé. Ce trait de personnalité est un des éléments qui caractérisent le profil consciencieux des individus (Costa et McCrae, 1992). Par définition, un individu consciencieux est déterminé, il possède un fort contrôle de lui-même et un besoin de rangement et de propreté important. Or, le juste-à-temps conduit à stocker les déchets dans 
des zones de vie, ce qui peut être perçu comme mal rangé, et à même le sol, ce qui peut être considéré comme sale : "si c'était du carrelage ça me gênerait moins de mettre mon sac poubelle non recyclable,... Or là sur du parquet, je... ça je supporte pas ça. Je trouve que c'est pas sain, et puis même sur le parquet, parce que souvent ça coule, etc., c'est pas propre et tout, donc ça c'est hors de question! »(Boris). Cette présence spatiale des déchets induit également des problèmes esthétiques qui peuvent gêner certains individus qui ne souhaitent pas que leurs poubelles soient visibles : "après c'est une affaire d'esthétique aussi, j'ai pas envie d'avoir un immense carton visible dans la pièce. C'est un arbitrage entre esthétique, développement durable, propreté » (Boris). Enfin, elle peut poser des problèmes de sécurité, lorsque de jeunes enfants font partie du ménage : «j'ai un carton dans lequel je mets tout ce qui est recyclable, principalement du plastique, parce que le verre avec ma fille, c'est délicat. Donc le verre je suis un peu embêté, je sais pas trop où le mettre. J'essaie de le cacher un peu, ou alors je le fais au fur et à mesure » (Boris). De plus, cette logistique conduit à se débarrasser fréquemment des déchets. Or, ceci peut poser problème aux individus qui ont tendance à tout garder (Guillard et Pinson, 2012) ou qui ont besoin de « refroidir » certains déchets avant de les jeter, comme c'est le cas des journaux (Bartiaux, 2002). Cette tendance peut être liée à la relation instrumentale qu'ils entretiennent avec certains objets et qui nourrit alors leur besoin de contrôle sur leur environnement (Richins, 1994) : "je les garde parce qu'habituellement... je suis quelqu'un qui adore bricoler, dessiner, tout ça. Et j'ai vu sur une revue un mec qui recyclait toutes ses bouteilles en plastique... il fait des supers trucs avec (...) au début j'en avais gardé quelques-unes en me disant c'est super pratique, j'ai toujours besoin d'une bouteille d'eau, et puis après j'ai vu ça, et je me suis dit "ça peut être pas mal" (...) j'aimerais bien essayer d'en faire quelque chose» (Clara). Enfin, les individus qui ont tendance à procrastiner (Darpy, 2002), c'est-à-dire à systématiquement reporter leurs décisions «à plus tard (Lay, 1986 ; Ferrari, 1991), sont également moins enclins à choisir cette logistique car elle implique une présence psychique forte des déchets. Des conflits peuvent donc se produire entre ces variables individuelles et le choix du juste-à-temps.

Un troisième type de logistique du tri : la «mutualisation»

Des flux de tri mutualisés avec d'autres flux logistiques du quotidien

La troisième logistique du tri est la mutualisation. Elle consiste à se servir des autres logistiques du quotidien, notamment les courses alimentaires, pour gérer de manière optimale les flux de déchets. Les déchets à recycler sont ainsi stockés dans le contenant qui sert à faire les courses (caddie, cabas), généralement placé dans la cuisine. Ce contenant est donc « rempli » des déchets à recycler (flux sortant) puis « vidé » des courses au retour au domicile 
(flux entrant). S'il n'est pas encore nécessaire de faire les courses, les individus utilisent fréquemment des stratégies de compactage des déchets pour éviter que le contenant ne déborde. Ceci conduit alors à un stockage d'un volume de déchets important au sein du contenant. Les individus peuvent également tirer parti d'autres activités logistiques que les courses, comme celles liées au transport des enfants (trajets pour l'école ou le parc, par exemple) : "j'y vais en voiture. Parce que c'est pas hyper loin mais c'est genre à 300-400 mètres, en général j'ai un gros sac donc c'est un peu encombrant donc je préfère y aller en voiture, et puis je me débrouille pour que ça cö̈ncide avec un trajet pour aller faire des courses ou déposer ma fille à la crèche, enfin un trajet où j’ai besoin de ma voiture (...) dans ma cuisine j'ai un petit coin dans lequel j'ai mis un grand sac, le cabas justement que je prends pour aller au recyclage puis faire mes courses »(Justine). Enfin, la mutualisation consiste non seulement à profiter d'autres flux logistiques du quotidien mais aussi des flux de déchets d'autres consommateurs. Ainsi, un répondant déclare récupérer les déchets organiques de proches vivant en appartement, les stocker temporairement à son domicile, puis les expédier dans sa résidence secondaire où le compost est utilisé : « ma mère met de côté ses épluchures de fruits, légumes, etc., qu'elle a stockées dans la cuisine, moi je les récupère et je les stocke ensuite chez moi avant de les amener sur mon terrain le week-end. On s'est organisé comme ça»(Cathy). La première forme de mutualisation consiste ainsi à faire du multidrop : un seul individu livre plusieurs points (points de collecte et école, par exemple) alors que la seconde relève du multipick: plusieurs individus fournissent un seul point (Le Moigne, 2013). Pour optimiser la logistique du tri, une collaboration avec des personnes hors du foyer apparaît alors opportune.

\section{Utiliser d'autres flux logistiques face à la double contrainte de la supply chain du tri} Cette logistique est déployée lorsque les individus estiment qu'ils ne peuvent tirer parti ni des possibilités de stockage en interne, ni des facilités du type de système de collecte proposé, en externe. Cette logistique se rencontre donc quand les individus font face à une supply chain globalement peu favorable qui limite selon eux la capacité à massifier et à expédier. C'est par exemple le cas des petites habitations situées soit en étage élevé en centre-ville soit dans des zones rurales et éloignées des points de collecte : "j’habite dans une tour de dix-neuf étages, au $15^{\text {ème }}$ (...) En bas de la tour quand on descend, mais vraiment à vingt mètres, y a un endroit pour collecter les verres et un endroit pour mettre tout ce qui est plastique (...) Mais du coup, je ne descendrais pas uniquement pour vider les poubelles, parce que je suis au $15^{\text {ème }}$, donc il faut attendre l'ascenseur, le prendre, descendre et sortir (...). Mais comme j'ai pas énormément de place dans ma cuisine, du coup je mets mes déchets dans des grands sacs 
plastiques, parce qu'avec des sacs tu peux plus facilement faire tes courses après »(Antoine).

Une mise à distance des déchets relativement faible

Cette logistique induit une mise à distance faible des déchets (Pierre, 2002). Ils ont tout d'abord une présence physique indéniable relative aux nombreux contacts nécessaires pour les trier. En effet, il faut non seulement les manipuler aux points de collecte, mais parfois aussi les laver, afin d'éviter qu'ils ne salissent le contenant utilisé pour les courses : "pour les bouteilles de vin, je rince avec de l'eau pour qu'il ne reste pas de vin qui pourrait couler et salir (...) D'ailleurs c'est arrivé plusieurs fois d'avoir des traînées qui collent et après il faut nettoyer» (Antoine). Cette présence physique des déchets est d'ailleurs rappelée au moindre point de blocage qui vient perturber une logistique totalement optimisée : «il y a des choses qui m'énervent c'est quand mon point de collecte habituel, là celui dont j'ai parlé, près de l'école, il est plein, quand le bac est plein ça, ça m'agace (...) je pars à la recherche d'une autre benne, enfin d'un autre point de recyclage pour jeter mes trucs, parce que je ne veux pas le mettre dans la poubelle classique»(Justine). Toutefois, parce que les individus stockent leurs déchets dans des contenants directement transportables, ils évitent de multiplier les manipulations : ils suivent alors un mode de préparation au domicile de type pick and pack (Mocellin, 2011). En outre, les déchets sont relativement présents spatialement. L'usage du contenant dédié aux courses atténue les problèmes esthétiques et de sécurité mais ne les élimine pas totalement, les déchets restant accessibles. Enfin, la présence psychique des déchets reste, elle aussi, relativement importante car il faut s'organiser et penser à les déposer. Des tactiques visant à éviter l'oubli sont alors mises en place, comme le stockage des poubelles devant la porte d'entrée. Cette logistique a cependant l'avantage d'intégrer les déchets dans des routines quotidiennes : "je l'ai intégré, voilà c'est juste un mini détour, enfin c'est pas un détour, c'est juste qu'il faut que je m'arrête [au point de collecte]» (Justine). Dans cette logistique, les individus vivent avec leurs déchets, au domicile et au cours de leurs déplacements, et considèrent qu'ils font partie de leur quotidien : " je ne trouve pas que ça soit des déchets sales, les ordures ménagères (...). Je ne trouve pas ça sale et je trouve que ça fait partie de la consommation. C'est un peu comme sur le plan physiologique on pourrait dire, on mange et après on évacue, c'est pareil »(Martine).

\section{Une logistique inadaptée aux individus mal organisés}

Cette logistique implique pour les individus d'être organisés et méticuleux : il faut trouver une place pour le contenant qui sert à stocker les déchets, le remplir en ayant parfois rincé les déchets au préalable, penser à ne pas l'oublier lors des déplacements, etc. Ce caractère 
méthodique fait référence à d'autres dimensions du besoin d'ordre des individus (Costa et McCrae, 1992) : ce ne sont plus simplement le rangement et la propreté qui sont importants mais aussi une capacité générale à savoir s'organiser, tant d'un point de vue spatial que temporel. Ainsi, les individus mal organisés ne sont pas enclins à opter pour cette logistique qui demande de la rigueur : "je trouve que le service de ramassage sur le trottoir dans ma résidence principale, ou ici dans les conteneurs mis à disposition dans la commune sont ni contraignants, ni pas contraignants, il faut y penser, il faut s'organiser (...) il m'est arrivé de partir de cette maison déjà deux fois dans les mois passés en oubliant le sac au milieu de la cuisine, parce que je suis parti, je ne l'ai pas vu, alors que je l'avais préparé... »(Clément).

Ces trois logistiques sont schématisées dans la Figure 1. Les activités physiques de chaque logistique sont illustrées en Annexe A4.

\section{< Insérer Figure 1 >}

\section{CONTRIBUTIONS THEORIQUES}

Nos résultats contribuent à la littérature à deux niveaux théoriques : (1) ils enrichissent la littérature en marketing sur le comportement de tri, qui ne se réduit pas à un niveau de tri (objectif à atteindre) mais comprend également toute une logistique (activités à réaliser) ; (2) ils contribuent au courant de la consumer logistics grâce à une meilleure caractérisation des logistiques déployées par les individus au cours d'une étape de la consommation, le tri.

\section{Le comportement de tri : un niveau de tri et une logistique du tri}

Notre recherche apporte une contribution théorique à la littérature sur le tri. Cherchant à expliquer le niveau de tri, la littérature en marketing s'est focalisée sur le rôle des motivations liées à des facteurs internes (attitude environnementale, croyances, valeurs) et externes (normes, incitations). Elle a également mis en évidence l'influence de variables contextuelles liées au domicile et au système de collecte proposé. La littérature fait ainsi globalement état d'un individu qui trie par motivation et du fait d'un contexte qu'il juge favorable. Notre recherche montre que ces variables ne sont pas suffisantes pour étudier le comportement de tri. Nous arrivons à la conclusion que, pour trier, un individu doit avoir la capacité de mettre en place toute une logistique, comprenant des activités de séparation, stockage et expédition, et ce, en fonction des caractéristiques de sa supply chain interne et externe et du rapport souhaité aux déchets, tant sur le plan physique, spatial que psychique. Les consommateurs recourent ainsi, en fonction de ces caractéristiques, à un type de logistique du tri: la massification, le juste-à-temps ou la mutualisation. Nous soulignons que, lorsque l'individu ne peut opter pour une logistique adaptée à la fois à sa supply chain et au rapport souhaité aux 
déchets, un état de tension assimilable à une forme de dissonance cognitive (Festinger, 1957) peut se créer. Dans une telle situation, l'individu trieur n'a souvent d'autre choix que d'optimiser un seul aspect : la supply chain, comme le fait Boris qui conserve le juste-à-temps malgré les problèmes d'esthétique et de sécurité qu'il évoque ; ou le rapport aux déchets, comme le fait Elodie qui préfère le juste-à-temps à la mutualisation pour satisfaire son besoin de propreté. La Figure 2 récapitule les variables qui influencent le choix d'un type de logistique.

\section{< Insérer Figure 2 >}

En caractérisant la logistique du tri, notre recherche fait écho à la proposition de Thøgersen (1997) qui considère un modèle de type MOA (motivation, opportunité et capacité) pour comprendre le comportement de tri. Si les motivations et les opportunités sont déterminantes pour trier, les capacités le sont également. C'est sur ces capacités, en particulier logistiques, que cette recherche s'est penchée. Elle montre que certains individus trient, même quand ils font face à une supply chain qu'ils jugent peu favorable en externe et/ou en interne. Ceci semble lié à leur capacité à adapter leur logistique en fonction de leur supply chain, afin d'atténuer son influence éventuellement défavorable. Inversement, une des raisons pour laquelle certains individus ne trient pas pourrait résider dans leur incapacité à déployer une logistique adaptée à leurs paramètres contextuels et individuels. Ceci est cohérent avec le fait que la gestion des déchets est un ensemble de micro-décisions ou micro-pratiques, qui font apparaître les «rugosités » du quotidien et les contraintes qui structurent la vie domestique (Desjeux, 2002). Cette recherche fournit donc une meilleure compréhension du comportement de tri : celui-ci étant composé d'un niveau de tri (ce que la littérature a essentiellement analysé jusqu'à présent) et d'une logistique (ce que cette recherche a caractérisé).

\section{Les logistiques des consommateurs : similarités et différences avec celles des entreprises}

En caractérisant les logistiques déployées par les individus durant cette étape de consommation qu'est le tri, notre recherche contribue au courant de la consumer logistics (Granzin et Bahn, 1989 ; Granzin, Painter et Valentin, 1997 ; Granzin, Painter et Bahn, 2005 ; Teller, Kotzab et Grant, 2012). De ce point de vue, notre caractérisation approfondit l'intuition de Granzin et Bahn (1989) selon laquelle les logistiques déployées par les consommateurs présentent à la fois des similitudes et des différences avec celles des entreprises. Ainsi, chacun des types de logistique du tri repose sur des principes de pilotage des flux physiques utilisés par les entreprises et qui ont été théorisés depuis plusieurs années 
par la littérature en logistique et en supply chain management. La massification renvoie ainsi à un principe de flux «poussés » qui conduit à un stockage important des produits et permet de développer une supply chain efficiente, c'est-à-dire visant avant tout à diminuer les coûts (Fisher, 1997). Inversement, le juste-à-temps s'apparente au principe de flux «tirés » (Ohno, 1988) qui conduit à une réduction des délais et à une accélération des flux permettant de mettre en place une supply chain réactive (Fisher, 1997). Enfin, la mutualisation avec d'autres consommateurs fait écho au principe «d'externalisation» des flux (Fulconis, Paché et Roveillo, 2011) qui conduit les entreprises à confier la réalisation de leurs activités logistiques à un même prestataire de service logistique et qui permet la mise en place d'une supply chain collaborative.

Nos résultats suggèrent cependant que les individus n'appliquent pas tels quels les principes logistiques des entreprises, et ce pour au moins trois raisons. La première tient au rapport qu'ils entretiennent avec les flux physiques durant la consommation. Alors que les entreprises voient dans les flux physiques de simples unités logistiques aux caractéristiques objectives (fragilité, poids, taille, etc.) qu'il s'agit de gérer rationnellement en fonction d'objectifs de coût et de service (Paché et Colin, 2000), tel n'est pas le cas des individus qui accordent de l'importance au type de rapport aux déchets. Les logistiques déployées par les consommateurs sont donc dictées non seulement par des principes rationnels, mais aussi sensoriels, comme en témoigne par exemple le dégoût lié aux odeurs ou à la saleté des déchets. La deuxième raison tient aux ressources limitées des individus. Alors que les entreprises font en sorte d'adapter leurs ressources à la logistique qu'elles mettent en place, en recourant notamment à des prestataires de services logistiques (Fulconis, Paché et Roveilllo, 2011), les consommateurs adaptent plutôt leur logistique à leurs ressources. Nos résultats confirment, comme l'avaient identifié Granzin et Bahn (1989), que le domicile est une ressource physique qui détermine fortement la logistique des consommateurs. Ils attestent aussi du rôle majeur des ressources physiques des individus eux-mêmes, certaines logistiques comme la massification pouvant être physiquement compliquées à adopter pour certains. Sur cette question des ressources, les moyens de transport dont disposent les individus influent également sur les logistiques retenues. Une troisième raison tient enfin au caractère plus informel des logistiques des consommateurs. Si elles ont toujours une part informelle (Crozier et Friedberg, 1977), les logistiques des entreprises s'appuient sur de nombreux dispositifs formalisés : des systèmes d'information pour piloter les flux (Fabbe-Costes, 2001), des fonctions logistiques institutionnalisées dans la structure de l'entreprise (Fabbe-Costes et Meschi, 2000), des indicateurs pour mesurer la performance logistique, etc. A la différence des entreprises, les 
individus font davantage appel à leur créativité et s'appuient sur des micro-pratiques : compactage des déchets, gerbage de plusieurs contenants, stockage vertical... (Annexe A4). Par exemple, un consommateur qui trie ses déchets en juste-à-temps ne dispose pas d'outils de pilotage formalisés et ne fait notamment pas appel à un système d'étiquettes ou de kanbans (Ohno, 1978) mais s'appuie plutôt sur une coordination informelle.

\section{IMPLICATIONS MANAGERIALES}

A l'heure où un enjeu sociétal fondamental est d'augmenter la contribution des individus au recyclage, il est important de considérer les implications managériales et sociétales de notre recherche. Jusqu'à présent, les acteurs institutionnels chargés d'inciter les consommateurs au tri (collectivités locales, syndicats intercommunaux, ADEME, Etat) ont développé deux types d'actions : (1) des actions visant à motiver les consommateurs au tri (par exemple, campagnes de communication sur l'importance du recyclage ; paiement des déchets au poids); (2) des actions visant à informer les consommateurs des règles du tri (par exemple, dépliants/applications indiquant quel type de déchet mettre dans quelle poubelle; informations sur les horaires et dates de collecte). De manière complémentaire à ces démarches, notre recherche suggère de développer un troisième type d'actions visant à accompagner les consommateurs dans l'amélioration de leurs capacités logistiques. Une telle approche rejoint le courant actuel du marketing collaboratif (Cova et Cova, 2009) qui souligne l'importance de former, éduquer et socialiser les consommateurs (Fisher, 1986; Goudarzi et Eiglier, 2006) aux tâches nécessaires à la consommation. L'enjeu semble ici de faire du consommateur un véritable « acteur » (Rouquet, Reniou et Goudarzi, 2013) au sein de la supply chain «inversée» (Lambert et Stock, 1981; Stock, 1992 ; Dowlatshahi, 2000 ; Rogers et Tibben-Lembke, 2001 ; Guide et Van Wassenhowe, 2002), c'est-à-dire du système inversé mis en place par les communes afin de collecter les déchets. Sa participation est indispensable afin d'interconnecter les supply chains classiques et inversées et de créer des supply chains en boucle fermée (Savaskan, Bhattacharya et van Wassenhove, 2004) au sein desquelles les matières peuvent être réutilisées, à des fins d'économie circulaire (Lévy, 2010). Pour développer les capacités logistiques des consommateurs et les rapprocher de celles des entreprises, trois pistes sont suggérées : l'amélioration du rapport aux déchets ; l'adaptation des ressources logistiques nécessaires au tri ; et la communication face au caractère informel de la logistique du tri. Tout d'abord, le fait d'entretenir un rapport trop proche aux déchets pouvant poser problème à certains individus, des démarches permettant leur mise à distance paraissent pertinentes. Au niveau du flux entrant, l'encombrement des déchets constituant une 
contrainte physique, la réduction du volume des déchets entrant dans les domiciles pourrait être encouragée. Dans ce cadre, les incitations à l'achat de produits avec moins d'emballage (Monnot et Reniou, 2012), compactables ou rechargeables pourraient être poursuivies par les pouvoirs publics et les industriels (par exemple sous la forme de mentions apposées sur les produits, à la manière des mentions «made in France »). Au niveau du flux sortant, des systèmes de collecte plus hygiéniques pourraient être conçus pour répondre au besoin de propreté de certains individus (par exemple en fournissant des conteneurs munis de trappes s'ouvrant avec les pieds plutôt qu'avec les mains).

Ensuite, en ce qui concerne les ressources logistiques, plusieurs suggestions peuvent être proposées, en particulier pour minimiser les efforts de stockage et d'expédition des déchets. Les recherches précédentes ont montré l'importance de faciliter le système de collecte pour encourager le tri (par l'augmentation du nombre de points de collecte, des fréquences de ramassage, etc.) car plus les ressources externes sont importantes, moins les consommateurs sont dépendants de leurs propres ressources, ce qui facilite leur logistique. Notre recherche montre toutefois que l'enjeu se situe aussi au niveau des ressources internes, c'est-à-dire du domicile lui-même. Elle souligne notamment que les bacs de tri constituent une ressource interne clef qui doit être adaptée en fonction du type de logistique retenue par les consommateurs. Dans ce cadre, les acteurs de la gestion des déchets pourraient équiper les individus en leur fournissant des bacs de tri individuels. Ceux-ci seraient variables en termes de taille pour s'adapter au contexte et pourraient être «deux-en-un », c'est-à-dire permettant à la fois le stockage et le transport des déchets afin de limiter les opérations de dépotage/rempotage. A un autre niveau, cette question des ressources pourrait être intégrée dans la construction des programmes immobiliers collectifs respectant les normes HQE (Haute Qualité Environnementale). L'éloignement du point de collecte et la taille de l'espace de stockage constituant des contraintes pour certains individus, l'idée serait de réduire les efforts logistiques en optant pour une gestion en flux «tendus »: dès que les déchets recyclables arriveraient au domicile, le trieur n'aurait qu'à les déposer dans des réceptacles dédiés, directement accessibles depuis son appartement (à la manière de « vide-ordures » qui seraient conçus avec des matériaux permettant d'éviter les nuisances sonores et hygiéniques), supprimant ainsi le stockage et l'expédition. Enfin, pour aller dans le sens d'une supply chain en boucle fermée, il serait intéressant d'étudier la faisabilité de mutualiser les logistiques de consommation des courses et du tri en impliquant un autre maillon de la chaîne: les distributeurs. Dans le cadre du développement de la livraison des courses à domicile, ces derniers pourraient éviter de repartir à vide après la livraison en mettant en place un système 
de récupération des déchets recyclables. Ceci permettrait aux consommateurs de ne plus avoir à assurer l'activité d'expédition aux points de collecte, les déchets étant directement prélevés à leur domicile.

Enfin, le caractère informel des logistiques des individus conduit à rappeler l'importance de la communication. De manière générale, la mise en place de spécialistes logistiques dans les communes pourrait être étudiée pour répondre aux questions des individus et les accompagner dans le déploiement d'une logistique qui leur convienne. Cette piste va plus loin que celle de Hopper et Nielsen (1991) qui suggèrent de désigner des ambassadeurs du tri par quartier. Elle pose néanmoins la question du coût de la démarche et des acteurs auxquels incomberait la responsabilité de ce coût. Des référentiels décrivant et expliquant les différents types de logistiques envisageables suivant le contexte pourraient constituer une alternative. Ces référentiels pourraient faire mention des différentes micro-pratiques utilisables pour réaliser la logistique du tri et viendraient compléter ceux qui existent déjà sur les pratiques de tri mais qui visent une diminution des efforts cognitifs plutôt que des efforts physiques. Ceci encouragerait probablement la créativité des individus dans leurs pratiques de tri.

\section{PERSPECTIVES DE RECHERCHE}

En dépit de ses contributions, cette recherche n'est pas exempte de limites qui peuvent donner lieu à des voies de recherche futures.

Premièrement, notre recherche, qui apporte des contributions à deux champs de littérature en marketing, celui du comportement de tri et celui de la consumer logistics, pourrait donner naissance à des recherches complémentaires. Au niveau de la littérature sur le tri, cette recherche a mis à jour trois types de logistique du tri qui peuvent être vus comme des idéauxtypes au sens de Weber (1965), c'est-à-dire comme une reconstruction de la réalité à partir des critères dégagés par les chercheurs sur le terrain. Ces idéaux-types, parce qu'ils se fondent sur des concepts préalablement identifiés (supply chains interne et externe) et sont mis en relation les uns avec les autres, constituent une typologie qui est en soi un apport théorique clef (Doty et Glick, 1994) pour la littérature sur le comportement de tri. Toutefois, il serait intéressant de construire un modèle intégrateur regroupant l'ensemble des variables qui influent sur ce comportement. Ceci n'est cependant pas sans poser des difficultés liées aux multiples influences qui existent entre les différentes variables. Il faudrait alors s'interroger sur le rôle respectif, au sein de ce modèle, des variables individuelles, des motivations, du contexte et de la capacité logistique des individus. De plus, l'influence de variables situationnelles devrait être prise en compte, les changements de situation (naissance, 
déménagement, etc.) pouvant influencer le choix d'un type de logistique. Au niveau de la littérature sur la consumer logistics, nous avons développé notre caractérisation en analysant une seule étape de la consommation, le tri. Or, il serait intéressant d'analyser d'autres logistiques de la consommation et de les mettre en parallèle avec nos résultats. Si notre recherche montre que la capacité logistique des individus est clef pour trier, l'est-elle également pour toutes les logistiques de la consommation? Les principes théoriques de la logistique d'entreprise sont-ils mobilisés par les consommateurs lorsqu'ils réalisent d'autres logistiques ? Il pourrait être intéressant de se focaliser sur le cas des courses par exemple (Antéblian et Barth, 2011) et d'identifier l'ensemble des étapes nécessaires au processus : décisions en termes de produits et quantités à acheter, moment d'achat, type de transport utilisé, magasin, emplacements de stockage au domicile, etc. Ceci permettrait de compléter les recherches déjà conduites sur l'étape du shopping présentées dans la revue de littérature.

Deuxièmement, malgré les efforts réalisés par les chercheurs pour déployer une méthodologie de qualité, cette recherche pourrait être améliorée sur le plan de sa validité interne. Les résultats seraient plus riches si des collectes de données sur des intervalles de temps plus longs étaient mises en place pour observer le processus. Ceci permettrait de réaliser des comptages précis pour chaque activité logistique sur une période donnée : temps consacré à la séparation des déchets et à leur expédition, volumes exacts de stockage, nombre de flux internes et externes, etc., et d'en faire une représentation visuelle grâce à un chronogramme, par exemple. A défaut de pouvoir mettre en place un tel dispositif méthodologique, la technique du journal de bord pourrait être envisagée et donnerait l'opportunité à plusieurs personnes du foyer d'y contribuer. Ceci serait opportun dans la mesure où la logistique du tri au sein d'un foyer composé de plusieurs personnes peut être une activité collective. L'aspect relatif à la répartition des tâches a été évoqué par certains répondants lors des entretiens mais n'a pas été approfondi dans cette recherche. Le tri pourrait être analysé à un niveau microsocial, c'est-à-dire intégrant les interactions entre individus au sein de foyer (Desjeux, 2004). Le tri fait en effet souvent l'objet d'une division sexuelle dans le ménage (Desjeux, 2002) qui reproduit les schémas de la domination masculine (Bourdieu, 1990) : aux femmes la gestion de l'intérieur du domicile et du stockage des déchets, aux hommes la gestion de l'extérieur et de l'expédition. Ceci conduirait à étendre l'unité d'analyse, de l'individu dans un lieu au foyer comprenant plusieurs individus. Cette prise en compte d'une double unité d'analyse est d'ailleurs répandue pour aborder des questions logistiques (Fabbe-Costes, Fulconis et Koulikoff-Souviron, 2013). En outre, le vécu et le sens des pratiques logistiques pour les répondants n'ont pas été approfondis dans cette recherche. Or, une piste de réflexion suggérée 
par ce travail est celle de la complexité perçue du tri. La logistique du tri ne semble en effet pas aller de soi, contrairement à ce que laisse penser la littérature sur les activités « ordinaires » (Dujarier, 2008 ; Benoit-Moreau, Bonnemaizon et Cadenat, 2013), car elle nécessite de coordonner un certain nombre d'activités. Néanmoins, nous n'avons pas dans nos données d'éléments relatifs à cette complexité perçue. D'autres recherches pourraient creuser ce sujet en adoptant un angle méthodologique plus interprétatif.

Troisièmement, la validité externe de la recherche pourrait être meilleure au moins sur deux aspects. D'une part, cette recherche s'est focalisée sur la logistique des déchets ménagers. Les conclusions pourraient être tout autres en étudiant des déchets aux caractéristiques physiques différentes, comme les encombrants qui relèvent d'autres logiques (Grygiel, 2002) : ils ne sont pas produits avec la même fréquence, n'ont pas les mêmes temps de stockage et de « refroidissement », et la relation affective avec eux est tout à fait différente (Belk, 1990 ; Guillard et Pinson, 2012), induisant des stéréotypes moteurs moins inconscients que pour les déchets ménagers (Hoarau, 1999). D’autre part, notre recherche a été réalisée dans un cadre culturel spécifique, celui de la France. Or, la France est un pays où il est accepté d'avoir des déchets dans sa cuisine, ce qui n'est pas le cas de la Chine ou de l'Inde, pays pour lesquels la mise à distance est évidente (Desjeux, 2002). Ainsi, il serait intéressant de répliquer cette recherche dans d'autres contextes culturels qui intègreraient notamment les normes et règlementations en matière de tri. En définitive, les types de système de collecte proposés par les communes, qui participent au développement durable de la société, visent à développer les contributions des individus au recyclage. Si les acteurs du tri tentent de confier un rôle important aux consommateurs, qui sont fortement générateurs de déchets, ils sont encore largement sous-exploités. L'enjeu reste de leur faire prendre conscience de l'importance de ce rôle et donc du sens qu'ils donnent à leur consommation (Özçağlar-Toulouse, 2009). 


\section{Annexes}

Annexe A1. Panorama des recherches sur les déterminants individuels du niveau de tri

\begin{tabular}{|c|c|c|}
\hline \multicolumn{3}{|c|}{ Rôle des caractéristiques sociodémographiques } \\
\hline \multirow{2}{*}{ Age } & $\begin{array}{l}\text { Les personnes âgées participent } \\
\text { plus au tri que les jeunes }\end{array}$ & $\begin{array}{l}\text { Vining et Ebreo (1990); Granzin et Olsen } \\
\text { (1991) }\end{array}$ \\
\hline & $\begin{array}{l}\text { Les effets de l'âge sont très } \\
\text { faibles }\end{array}$ & Shrum, Lowrey et McCarty (1994) \\
\hline \multirow{2}{*}{ Revenu } & $\begin{array}{l}\text { Le revenu est positivement corrélé } \\
\text { au tri }\end{array}$ & Vining et Ebreo (1990) ; Berger (1997) \\
\hline & $\begin{array}{l}\text { Il n'existe aucun lien entre revenu } \\
\text { et tri }\end{array}$ & Granzin et Olsen (1991) \\
\hline $\begin{array}{c}\text { Niveau } \\
\text { d'éducation }\end{array}$ & $\begin{array}{l}\text { Il n'existe pas de } \\
\text { significative entre le } \\
\text { d'éducation et le tri }\end{array}$ & $\begin{array}{l}\text { Granzin et Olsen (1991); Vining et Ebreo } \\
\text { (1990) }\end{array}$ \\
\hline Sexe & $\begin{array}{l}\text { Les femmes participent davantage } \\
\text { au tri dans le foyer }\end{array}$ & $\begin{array}{l}\text { Granzin et Olsen (1991); Stern et alii } \\
\text { (1993) ; Iyer et Kashyap (2007) }\end{array}$ \\
\hline \multicolumn{3}{|c|}{ Rôle des facteurs de motivation internes } \\
\hline \multirow{2}{*}{ Attitude } & $\begin{array}{l}\text { Une attitude favorable envers le } \\
\text { tri influence positivement le } \\
\text { comportement de tri }\end{array}$ & $\begin{array}{l}\text { McGuiness, Jones et Cole (1977); } \\
\text { McCarty et Shrum (1994); Knussen et alii } \\
(2004)\end{array}$ \\
\hline & $\begin{array}{l}\text { L'attitude environnementale n'a } \\
\text { pas de lien avec le comportement } \\
\text { de tri }\end{array}$ & $\begin{array}{l}\text { Vining et Ebreo (1990); Oskamp et alii } \\
\text { (1991); Gamba et Oskamp (1994); Iyer et } \\
\text { Kashyap (2007) }\end{array}$ \\
\hline Valeurs & $\begin{array}{l}\text { Les individualistes adoptent } \\
\text { davantage le tri mais les individus } \\
\text { sont tantôt collectivistes tantôt } \\
\text { individualistes }\end{array}$ & McCarty et Shrum (2001) \\
\hline \multicolumn{3}{|c|}{ Rôle des facteurs de motivation externes } \\
\hline \multirow[t]{2}{*}{$\begin{array}{l}\text { Normes } \\
\text { sociales }\end{array}$} & $\begin{array}{l}\text { Il existe une relation entre les } \\
\text { normes subjectives et le tri }\end{array}$ & $\begin{array}{l}\text { Vining et Ebreo (1990); Granzin et Olsen } \\
\text { (1991); Jackson et alii (1993); Bagozzi et } \\
\text { Dabholkar (1994); Taylor et Todd (1995); } \\
\text { Minton et Rose (1997); Pieters et alii } \\
\text { (1998) }\end{array}$ \\
\hline & $\begin{array}{l}\text { Il n'y a pas de relation entre les } \\
\text { normes subjectives et le tri }\end{array}$ & $\begin{array}{l}\text { Dahab, Gentry et } \mathrm{Su}(1995) \text {; Knussen et } \\
\text { alii (2004) }\end{array}$ \\
\hline $\begin{array}{l}\text { Incitations } \\
\text { financières }\end{array}$ & $\begin{array}{l}\text { Les incitations sont efficaces si } \\
\text { elles sont suffisamment } \\
\text { importantes sur le long terme }\end{array}$ & $\begin{array}{l}\text { Diamond et Loewy (1991); Iyer et } \\
\text { Kashyap (2007) }\end{array}$ \\
\hline Information & $\begin{array}{l}\text { Les individus les plus informés } \\
\text { adoptent davantage le tri mais il } \\
\text { existe plusieurs types } \\
\text { d'informations }\end{array}$ & Ellen (1994) ; Iyer et Kashyap (2007) \\
\hline
\end{tabular}


Annexe A2. Echantillon

\begin{tabular}{|c|c|c|c|c|c|c|c|c|c|}
\hline \multicolumn{3}{|c|}{ Identification } & \multicolumn{4}{|c|}{ Variables contextuelles } & \multicolumn{3}{|c|}{ Variables sociodémographiques } \\
\hline Cas & Prénom fictif & Niveau de tri & Type de logement & Type de zone & Lieu d'habitation & Système proposé & Âge & Profession & Nbre de pers. \\
\hline 1 & Antoine & Complet & Appartement & Urbaine & Bouches du Rhône (13) & Complet & 35 & Enseignant & 3 \\
\hline 2 & Boris & Partiel & Appartement T3 & Urbaine & Hauts-de-Seine (92) & Complet & 32 & Cadre & 3 \\
\hline 3 & Cathy & Complet & Appartement T5 & Urbaine & Seine Maritime (76) & Dépôt & 31 & Formatrice & 4 \\
\hline 4 & Célia & Partiel & Maison & Rurale & Vaucluse (84) & Dépôt & 65 & Retraité & 2 \\
\hline 5 & Célia & Partiel & Maison & Urbaine & Bouches du Rhône (13) & Dépôt & 65 & Retraité & 2 \\
\hline 6 & Clara & Partiel & Appartement T1 & Urbaine & Marne (51) & Partiel & 25 & Etudiante & 1 \\
\hline 7 & Clément & Complet & Maison & Urbaine & Seine et Marne (77) & Complet & 66 & Retraité & 1 \\
\hline 8 & Clément & Complet & Maison & Rurale & Isère (38) & Dépôt & 66 & Retraité & 1 \\
\hline 9 & Elodie & Complet & Appartement T3 & Urbaine & Hauts-de-Seine (92) & Complet & 30 & Enseignante & 2 \\
\hline 10 & Estelle & Partiel & Appartement T5 & Urbaine & Seine Maritime (76) & Dépôt & 55 & Au foyer & 2 \\
\hline 11 & Félix & Complet & Appartement T5 & Urbaine & Val d'Oise (95) & Dépôt & 35 & Consultant & 3 \\
\hline 12 & Hortense & Complet & Appartement T3 & Urbaine & Seine Maritime (76) & Complet & 84 & Retraité & 1 \\
\hline 13 & Justine & Complet & Appartement T4 & Rurale & Haute Savoie (74) & Dépôt & 32 & Au chômage & 4 \\
\hline 14 & Martine & Complet & Maison & Urbaine & Paris (75) & Partiel & 64 & Retraité & 2 \\
\hline 15 & Martine & Complet & Maison & Rurale & Lot (46) & Dépôt & 64 & Retraité & 2 \\
\hline 16 & Muriel & Complet & Appartement T4 & Urbaine & Hauts-de-Seine (92) & Complet & 87 & Retraité & 2 \\
\hline 17 & Nadine & Partiel & Maison & Urbaine & Hauts-de-Seine (92) & Partiel & 58 & Enseignante & 2 \\
\hline 18 & Nadine & Complet & Maison & Rurale & Gironde (33) & Complet & 58 & Expert-comptable & 2 \\
\hline 19 & Simon & Partiel & Appartement T2 & Urbaine & Paris (75) & Complet & 26 & Cadre & 1 \\
\hline 20 & Yolande & Complet & Maison & Urbaine & Haute-Garonne (31) & Dépôt & 31 & Mère au foyer & 3 \\
\hline
\end{tabular}


Annexe A3. Structure des données

Concepts de premier niveau

Thèmes de

Thèmes

deuxième niveau agrégés

\begin{tabular}{|l|l|l|}
\hline $\begin{array}{l}\text { Type de contenant utilisé } \\
\text { Lieu de réalisation de la séparation } \\
\text { Nombre de séparations réalisées }\end{array}$ & & $\begin{array}{c}\text { Activité de } \\
\text { séparation }\end{array}$ \\
\hline $\begin{array}{l}\text { Lieu de stockage } \\
\text { Capacité de stockage }\end{array}$ & & Activité de \\
Nombre d'étapes de stockage & & \\
\hline $\begin{array}{l}\text { Fréquence des expéditions } \\
\text { Nature dédiée/mutualisée de l'expédition } \\
\text { Facteur déclencheur de l'expédition } \\
\text { Type de préparation de l'expédition } \\
\text { Type de moyen de transport utilisé }\end{array}$ & & \\
\hline
\end{tabular}

Taille du domicile

Existence d'un local isolé au domicile

Taille et type d'aménagement de la cuisine

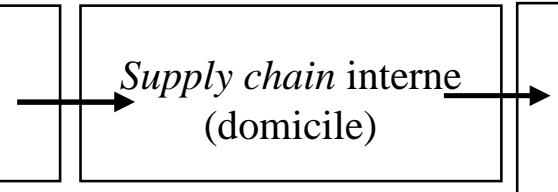

(domicile)

Type de collecte

Fréquence de collecte

Distance des conteneurs

Disponibilité des conteneurs

Commodité des conteneurs

Caractère visible du stockage

Caractère odorant du stockage

Caractère esthétique du stockage

Caractère préoccupant au quotidien

Caractère ordonné du stockage

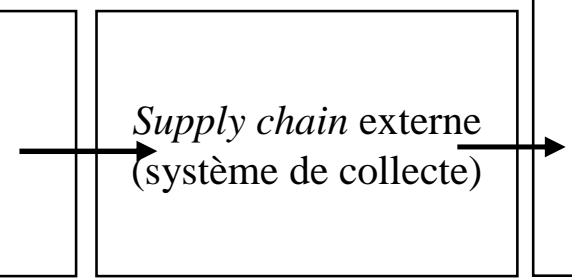

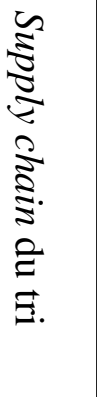

Poids et volume des déchets

Manipulations des déchets

Durée du stockage

Besoin d'ordre

Tendance à tout garder

Tendance à procrastiner

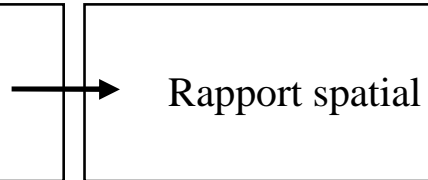

Capacités physiques de l'individu

Age

Sexe

Composition du ménage

Variables

physiques

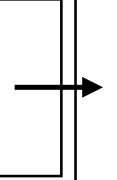

Rapport psychique
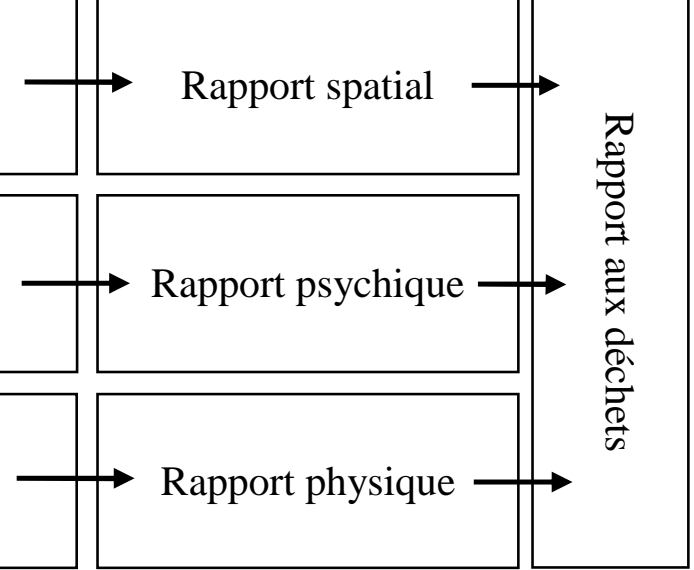
Annexe A4. Photographies illustratives des activités logistiques de chaque type de logistique du tri

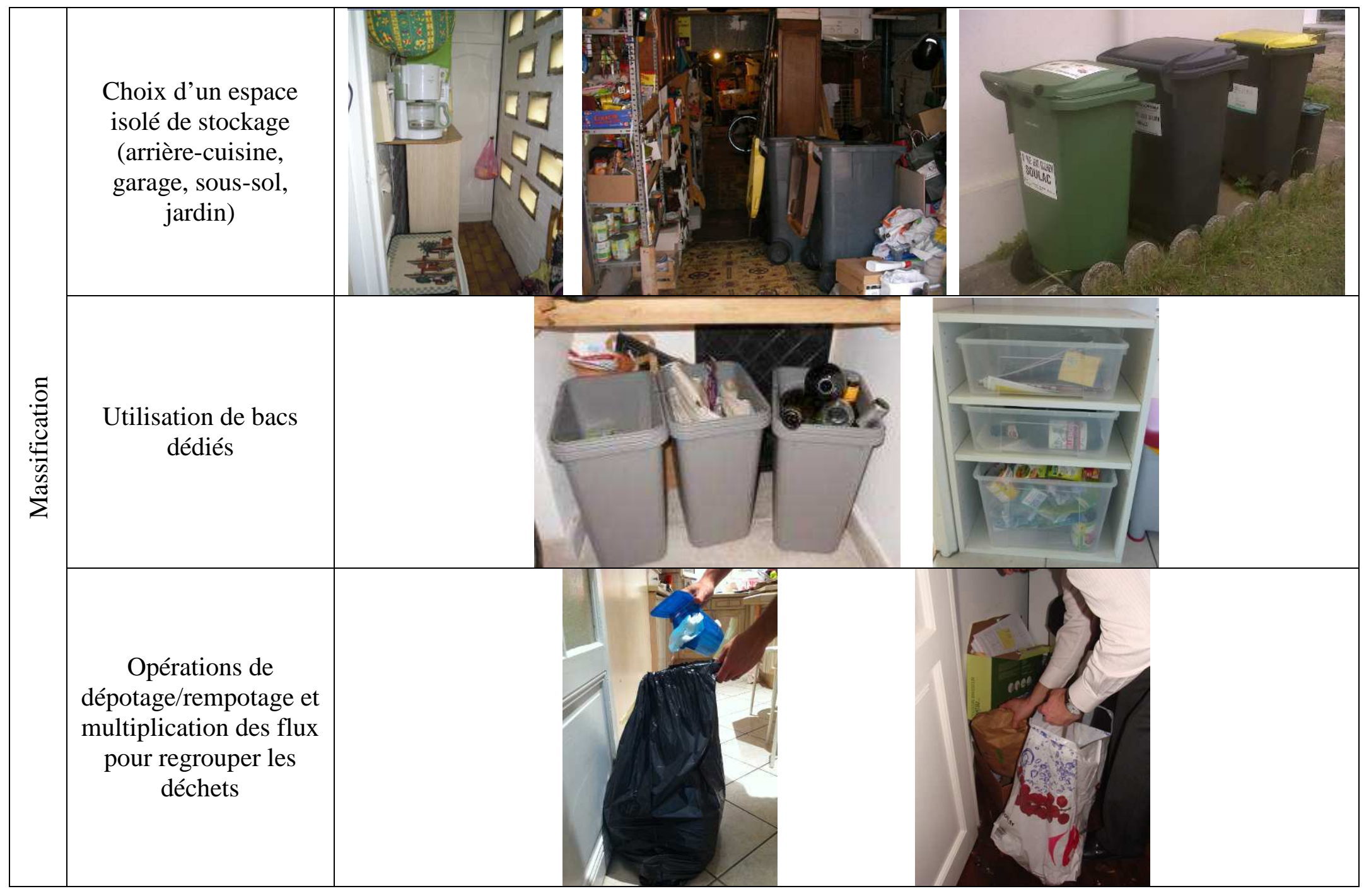




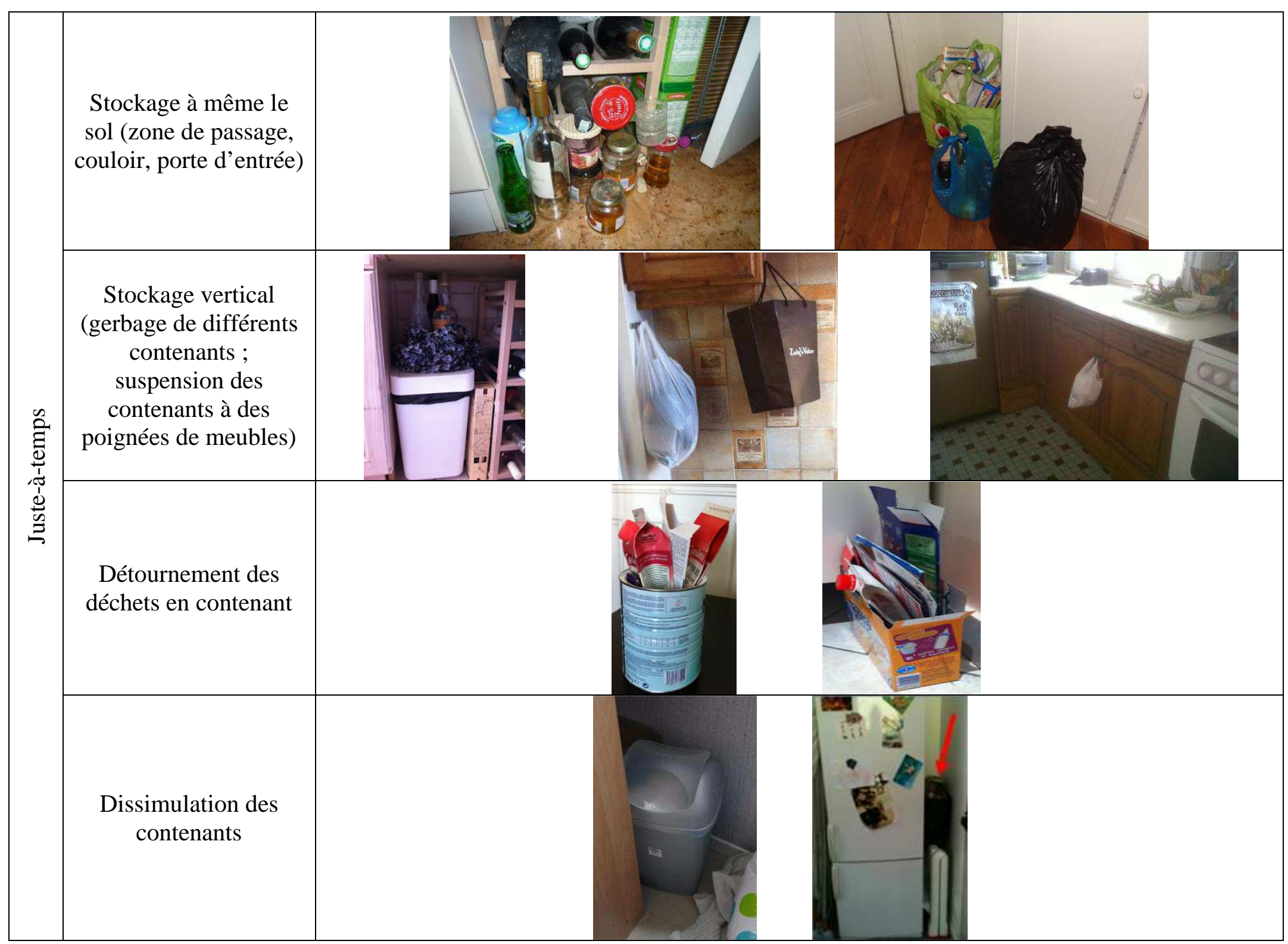




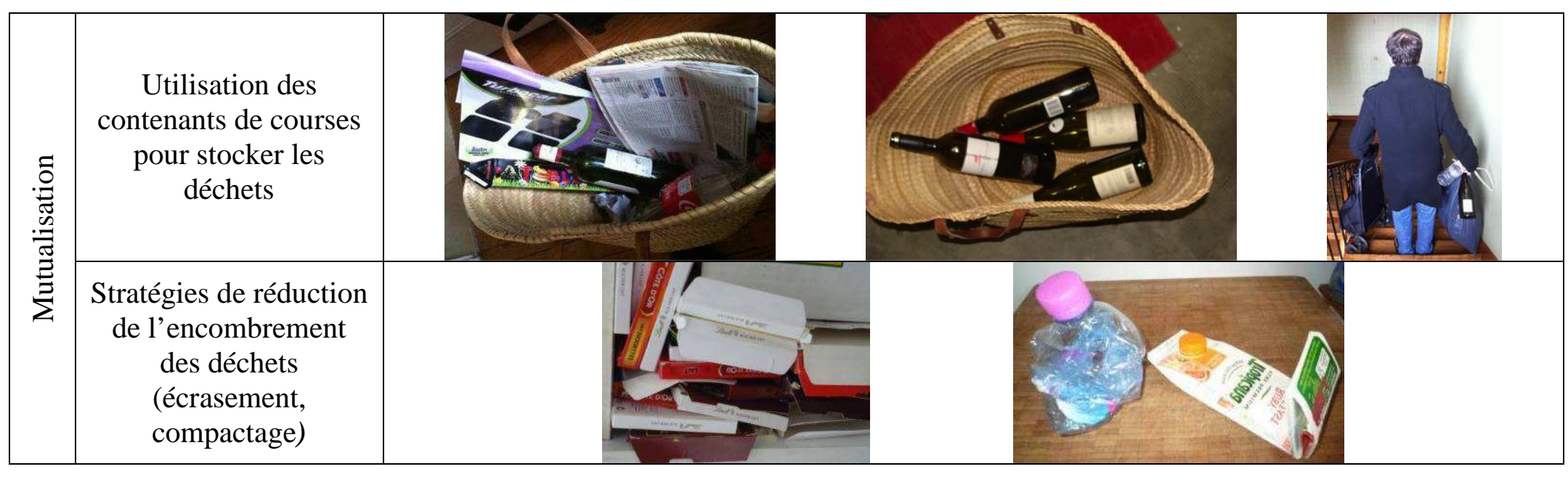




\section{Figures}
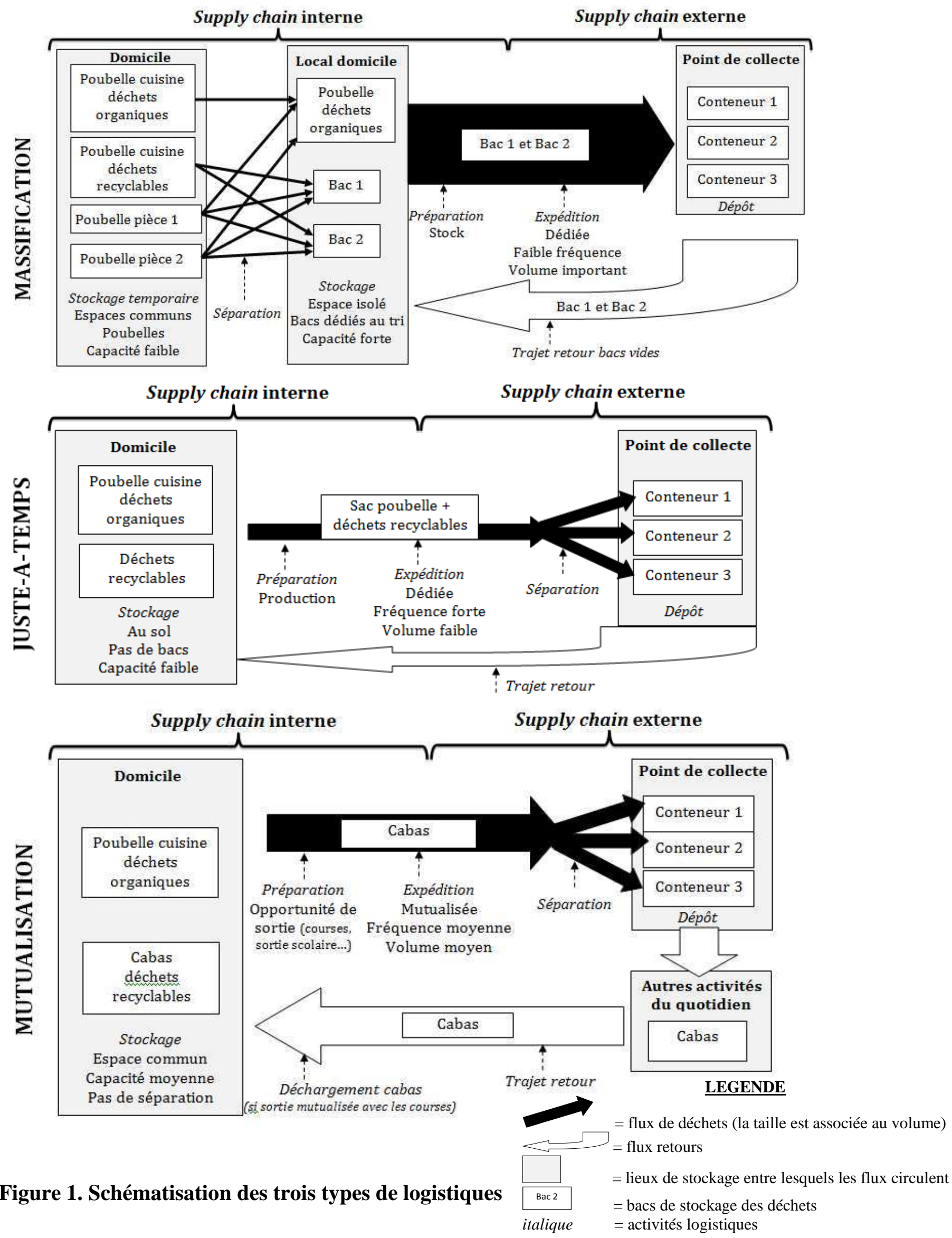


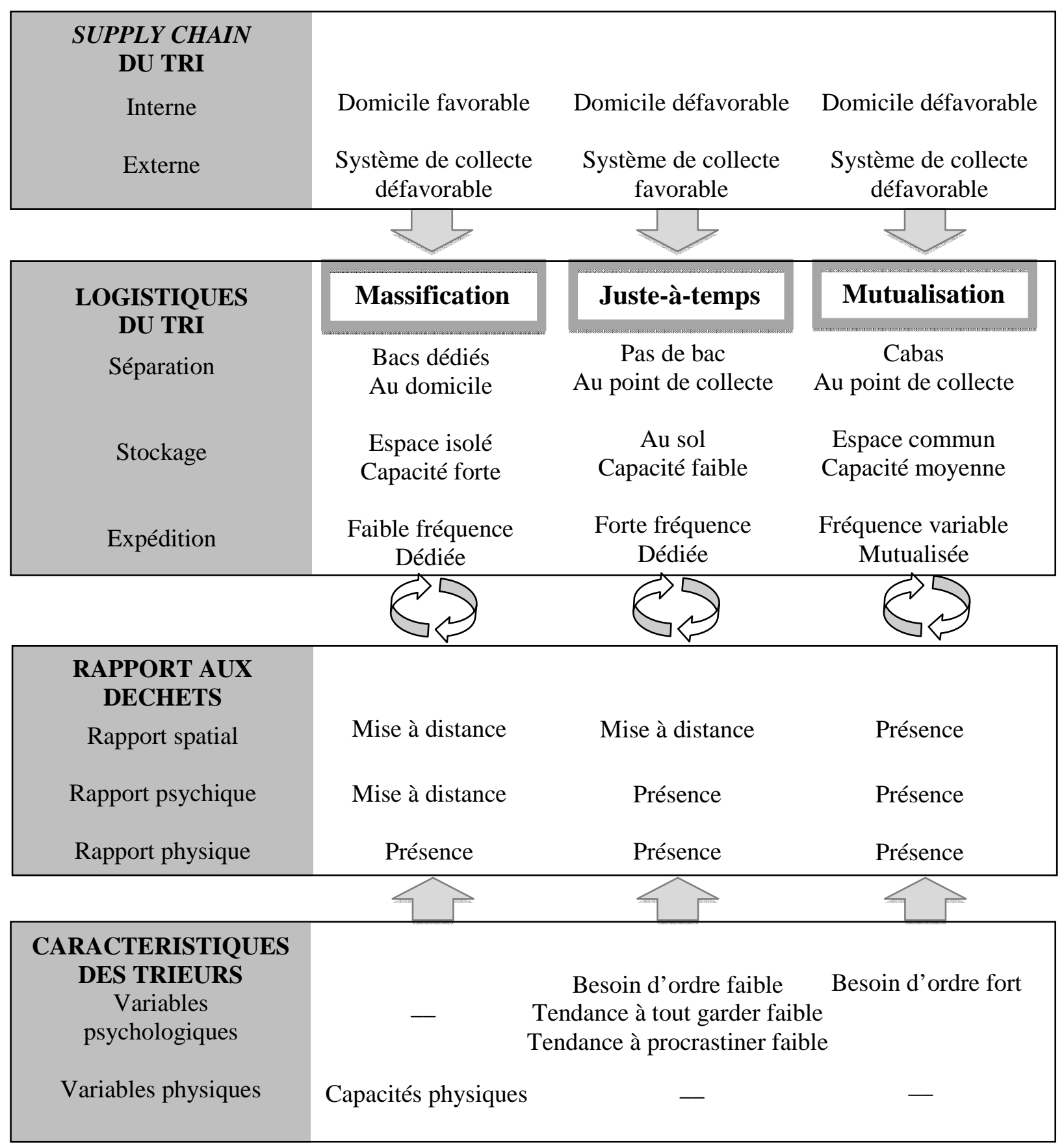

Figure 2. Variables influençant le choix d'un type de logistique du tri 


\section{Références bibliographiques}

ADEME (2012), http://www2.ademe.fr/servlet/KBaseShow? catid=12843\&p1=4\&p2=12551

Allard-Poesi F. (2003), Coder les données, in Y. Giordano (coord.) Conduire un projet de recherche, une perspective qualitative, Editions EMS (Management \& Société), 245-290.

Antéblian B. et Barth I. (2011), Les petites histoires extraordinaires des courses ordinaires : ethnographie des courses, EMS (Management \& Société).

Bagozzi R.P. et Dabholkar P.A. (1994), Consumer recycling goals and their effect on decisions to recycle: a means-end chain analysis, Psychology \& Marketing, 11, 4, 313-340.

Bartiaux F. (2002), Relégation et identité : les déchets domestiques et la sphère privée, in M. Pierre (coord), Les déchets ménagers, entre privé et public. Approches sociologiques, Paris, l'Harmattan, 123-146.

Bekin C., Carrigan M. et Szmigin I. (2007), Beyond recycling: "commons-friendly" waste reduction at new consumption communities, Journal of Consumer Behavior, 6, 5, 207-286.

Belk R.W. (1990), The role of possessions in constructing and maintaining a sense of past, Advances in Consumer Research, 17, 669-676.

Benoit-Moreau F., Bonnemaizon A. et Cadenat S. (2013), La participation ordinaire. Mieux comprendre le vécu du consommateur pour faciliter son adhésion, Revue Française de Gestion, 234, 151-166.

Berger I. (1997), The demographics of recycling and the structure of environmental behavior, Environment and Behavior, 28, 4, 515-531.

Biswas A., Licata J.W., McKee D., Pullig C. et Daughtridge C. (2000), The recycling cycle: an empirical examination of consumer waste recycling and recycling shopping behaviors, Journal of Public Policy and Marketing, 19, 1, 93-105.

Boldero J. (1995), The prediction of household recycling of newspapers: the role of attitudes, intentions, and situational factors, Journal of Applied Social Psychology, 25, 5, 440-462.

Bourdieu P. (1990). La domination masculine, Actes de la recherche en sciences sociales, 84, $1,2-31$.

Boyd T.C. et McConocha D.M. (1996), Consumer household materials and logistics management: inventory ownership cycle, The Journal of Consumer Affairs, 30, 1, 218-249.

Bowersox D.J (1978), Logistical management, $2^{\text {ème }}$ edition, New-York, McMillan.

Callon M. (1986), Eléments pour une sociologie de la traduction. La domestication des coquilles Saint-Jacques et des marins-pêcheurs dans la baie de Saint-Brieuc, L'année Sociologique, 36, 169-208. 
Christopher M. (2005), Logistics and Supply Chain Management: creating value-adding networks, $3^{\text {ème }}$ édition, Prentice-Hall, Harlow.

Costa P.T. et McCrae R.R. (1992), Manual of revised NEO personality inventory, Odessa, Psychological Assessment Resources.

Cova B. et Cova V. (2009), Les figures du nouveau consommateur: une genèse de la gouvernementalité du consommateur, Recherche et Application en Marketing, 24, 3, 81100.

Crozier M. et Friedberg E. (1977), L'acteur et le système, Paris, Seuil.

Dahab D.J., Gentry J.W. et Su W. (1995), New ways to reach non-recyclers: an extension of the model of reasoned action to recycling behaviors, Advances in Consumer Research, 22, 251-256.

Darpy D. (2002), Le report d'achat expliqué par le trait de procrastination et le potentiel de procrastination, Recherche et Applications en Marketing, 17, 2, 1-21.

Davies J., Foxall G.R. et Pallister J. (2002), Beyond the intention-behaviour mythology: an integrated model of recycling, Marketing Theory, 2, 1, 29-113.

Desjeux D. (2002), Les espaces sociaux du déchet: une microsociologie du quotidien encastrée dans le macrosocial, postface, in M. Pierre (coord), Les déchets ménagers, entre privé et public. Approches sociologiques, Paris, l'Harmattan, 173-181.

Desjeux D. (2004), Les sciences sociales, Paris, PUF.

De Young R. (1988/1989), Exploring the differences between recyclers and non-recyclers: the role of information, Journal of Environmental Systems, 18, 4, 341-351.

Diamond W.D. et Loewy B.Z. (1991), Effects of probabilistic rewards on recycling attitudes and behavior, Journal of Applied Social Psychology, 21, 19, 1509-1607.

Doty D.H. et Glick W.H. (1994), Typologies as a unique form of theory building: toward improved understanding and modeling, Academy of Management Review, 19, 2, 230-251.

Douglas M. (2001), De la souillure, Paris, La Découverte.

Dowlatshahi S. (2000), Developing a theory of reverse logistics, Interfaces, 30, 3, 143-155.

Dujarier M.-A. (2008), Le travail du consommateur. De McDo à eBay: comment nous coproduisons ce que nous achetons, Paris, La Découverte.

Ellen P.S. (1994), Do we know what we need to know? Objective and subjective knowledge effects on pro-environmental behaviors, Journal of Business Research, 30, 1, 43-52.

Fabbe-Costes N. et Meschi P.-X. (2000), Situations-types et évolutions de la place de la logistique dans l'organisation, Logistique et Management, 8, 1, 101-112. 
Fabbe-Costes N. (2001), Le pilotage des supply chains: un défi pour les systèmes d'information et de communication logistiques, Gestion 2000, 19, 1, 75-92.

Fabbe-Costes N., Fulconis F. et Koulikoff-Souviron M. (2013), La recherche en management logistique : la question des unités d'analyse et du périmètre, in N. Fabbe-Costes et G. Paché (coord.), La logistique: une approche innovante des organisations, Aix-enProvence, Presses Universitaires de Provence, 49-60.

Ferrari J.R. (1991), Compulsive procrastination: some self-reported characteristics, Psychological Reports, 68, 445-458.

Festinger L. (1957), A theory of cognitive dissonance, Stanford University Press.

Fiorello A. (2011), Le comportement de tri des déchets ménagers : une approche marketing, Thèse de Doctorat en Sciences de Gestion, Université de Nice-Sophia Antipolis, France.

Fisher C.D. (1986), Organizational socialization: an integrative review, Research in Personnel and Human Resources Management, 4, 101-145.

Fisher M.L. (1997), What is the right supply chain for your product?, Harvard Business Review, 75, 2, 105-116.

Fulconis F, Paché G. et Roveillo G. (2011), La prestation de service logistique. Origines, enjeux et perspectives, EMS (Management \& Société).

Gamba R.J. et Oskamp S. (1994), Factors influencing community residents' participation in commingled curbside recycling programs, Environment and Behavior, 26, 587-612.

Goudarzi K. et Eiglier P. (2006), La socialisation organisationnelle du client dans les entreprises de service : concept et dimensions, Recherche et Application en Marketing, 21, $3,65-90$.

Goudarzi K. et Rouquet A. (2013), Les rôles des clients dans la logistique de distribution des produits, Décisions Marketing, 69, 111-116.

Granzin K.L. et Bahn K.D. (1989), Consumer logistics: conceptualization, pertinent issues and a proposed program for research, Journal of the Academy of Marketing Science, 17, 1, 91-101.

Granzin K.L. et Olsen J.E. (1991), Characterizing participants in activities protecting the environment: a focus on donating, recycling, and conservation behaviors, Journal of Public Policy \& Marketing, 10, 2, 1-27.

Granzin K.L., Painter J.J. et Valentin E.K. (1997), Consumer logistics as a basis for segmenting retail markets. An exploratory inquiry, Journal of Retailing and Consumer Services, 4, 2, 99-107. 
Granzin K.L., Painter J.J. et Bahn K.D. (2005), An empirical test of household's participation in the distribution supply chain process, Journal of Marketing Channels, 14, 4, 67-89.

Grenier C. et Josserand E. (1999), Recherches sur le contenu et recherches sur le processus, in R.-A. Thiétart (coord.), Méthodes de Recherche en Management, Paris, Dunod, 116-131.

Grygiel C. (2002), Des gestes pour l'oubli. Manipulation des déchets dans l'espace domestique, in M. Pierre (coord), Les déchets ménagers, entre privé et public. Approches sociologiques, Paris, l'Harmattan, 147-172.

Guide V.D.R. Jr. et Van Wassenhove L.N. (2002), The reverse supply chain, Harvard Business Review, 80, 2, 25-26.

Guillard V. et Pinson C. (2012), Comprendre et identifier les consommateurs qui ont tendance à « tout » garder, Recherche et Applications en Marketing, 27, 3, 57-79.

Heckler S.E. (1994), The role of memory in understanding and encouraging recycling behaviour, Psychology \& Marketing, 11, 4, 375-392.

Heskett J.L. (1977), Logistics. Essential to strategy, Harvard Business Review, 55, 6, 85-95.

Hoarau F. (1999), Trier, transporter à Emmaüs. Ethnographie, sens et sciences de l'action, in M.P. Julien et J.P. Warniers (coord.), Corps à corps avec l'objet. Approches de la culture matérielle, Paris, L'Harmattan.

Hopper J.R. et Nielsen J.M. (1991), Recycling as altruistic behavior: normative and behavioral strategies to expand participation in a community recycling program, Environment and Behavior, 23, 2, 195-220.

Hornik J. et Cherian J. (1995), Determinants of recycling behavior: a synthesis of research results, The Journal of Socio-Economics, 24, 1, 105-128.

Humphrey C.R., Bord R.J., Hammond M.M. et Mann S.H. (1977), Attitudes and conditions for cooperation in a paper recycling programs, Environment and Behavior, 9, 1, 107-124.

Iyer E.S. et Kashyap R.K. (2007), Consumer recycling: role of incentives, information and social class, Journal of Consumer Behavior, 6, 1, 32-47.

Jackson A.L., Olsen J.E., Granzin K.L. et Burns A.C. (1993), An investigation of determinants of recycling consumer behaviour, Advances in Consumer Research, 20, 1, 481-487.

Jolivet P. (1999), Le consommateur responsable ? Etude du cas des déchets ménagers, Humanisme et Entreprise, 238, 33-46.

Kaufmann J.-C. (1993), Sociologie du couple, Paris, PUF. 
Knussen C., Yule F., MacKenzie J. et Wells M. (2004), An analysis of intentions to recycle household waste: the roles of past behavior, perceived habit, and perceived lack of facilities, Journal of Environmental Psychology, 24, 2, 237-246.

Kolbe R.H. et Burnett M.S. (1991), Content-analysis research: an examination of applications with directives for improving research reliability and objectivity, Journal of Consumer Research, 18, 2, 243-250.

Kreziak D. et Cova V. (2010), "Ça peut toujours servir !" : le consommateur ferrailleur, Actes des $15^{\text {èmes }}$ Journées de Recherche en Marketing de Bourgogne, Dijon, France.

Lambert D.M. et Stock J.R. (1981), Strategic physical distribution management, Hollywood, Irwin.

Langley A. (1999), Strategies for theorizing from process data, The Academy of Management Review, 24, 4, 691-710.

Lay C.H. (1986), At last, my research article on procrastination, Journal of Research in Personality, 20, 4, 474-495.

Le Moigne R. (2013), Supply chain management. Achat, production, logistique, transport, vente, Paris, Dunod.

Lévy J-C. (2010), L'économie circulaire : l'urgence écologique ? Monde en transe, Chine en transit, Paris, Presses de l'Ecole nationale des Ponts \& Chaussées.

Lincoln Y. et Guba E. (1985), Naturalistic inquiry, Beverly Hills, CA: Sage.

McCarty J.A. et Shrum L.J. (1994), The recycling of solid wastes: personal values, value orientations, and attitudes about recycling as antecedents of recycling behaviour, Journal of Business Research, 30, 1, 53-62.

McCarty J.A. et Shrum L.J. (2001), The influence of individualism, collectivism, and locus of control on environmental beliefs and behavior, Journal of Public Policy and Marketing, $20,1,93-104$.

McGuinness J., Jones A.P. et Cole S.G. (1977), Attitudinal correlates of recycling behavior, Journal of Applied Psychology, 62, 4, 376-84.

Miles M.B. et Huberman A.M. (2003), Analyse des données qualitatives, $2^{\mathrm{ème}}$ édition, Bruxelles, De Boeck Université.

Minton A.P. et Rose R.L. (1997), The effects of environmental concern on environmentally friendly consumer behavior: an exploratory study, Journal of Business Research, 40, 1, 3748.

Mocellin F. (2011), Gestion des stocks et des magasins. Pratiques des méthodes logistiques adaptées au lean manufacturing, Paris, Dunod. 
Monnot E. et Reniou F. (2012), Les suremballages, des emballages superflus pour les consommateurs ?, Décisions Marketing, 65, 31-43.

Ohno T. (1988), Toyota production system: beyond large-scale production, London, Productivity Press.

Oskamp S., Harrington M.J., Edwards T.C., Sherwood D.L., Okuda S.M. et Swanson D.C. (1991), Factors influencing household recycling behavior, Environment and Behavior, 23, 4, 494-519.

Özçağlar-Toulouse N. (2009), Quel sens les consommateurs responsables donnent-ils à leur consommation? Une approche par les récits de vie, Recherche et Applications Marketing, 24, 3, 3-24.

Paché G. et Colin J. (2000), Recherche et applications en logistique : des questions d'hier, d'aujourd'hui et de demain, in N. Fabbe-Costes, J. Colin et G. Paché (coord.), Faire de la recherche en logistique et distribution, Paris, Vuibert, 31-53.

Pettigrew A.M. (1997), What is a processual analysis, Scandinavian, Journal of Management, $13,4,337-348$.

Pierre M. (2002), Objets regardés, déchets inventés : les poubelles, du seuil de l'appartement au seuil de l'immeuble, in M. Pierre (coord), Les déchets ménagers, entre privé et public. Approches sociologiques, Paris, l'Harmattan, 47-72.

Pieters R.G.M (1991), Changing garbage disposal patterns of consumers: motivation, ability, and performance, Journal of Public Policy and Marketing, 10, 2, 59-76.

Pieters R., Bijmolt T., van Raaij F. et de Kruijk M. (1998), Consumers' attributions of proenvironmental behavior, motivation, and ability to self and others, Journal of Public Policy and Marketing, 17, 2, 215-225.

Richins M.L. (1994), Valuing things: the public and private meanings of possessions, Journal of Consumer Research, 21, 4, 504-521.

Rogers D. et Tibben-Lembke R. (2001), An examination of reverse logistics practices, Journal of Business Logistics, 22, 2, 129-148.

Rouquet A., Reniou F. et Goudarzi K. (2013), Le client « acteur » de l'organisation : enjeux et perspectives pour les sciences de gestion, Revue Française de Gestion, 234, 5, 8598.

Rumpala Y. (1999), Le réajustement du rôle des populations dans la gestion des déchets ménagers. Du développement des politiques de collecte sélective à l'hétérorégulation de la sphère domestique, Revue française de science politique, 49, 4, 601-630.

Rychlak J. F. (1977), The psychology of rigorous humanism, New York, John Wiley Inc. 
Sampson S.E. (2000), Customer-supplier duality and bidirectional supply chains in service organizations, International Journal of Service Industry Management, 11, 4, 348-364.

Savaskan R.C., Bhattacharya S. et van Wassenhove L.N. (2004), Closed- loop supply chain models with product remanufacturing, Management Science, 50, 2, 239-252.

Shrum L.J., Lowrey T.M. et McCarty J.A. (1994), Recycling as a marketing problem: a framework for strategy development, Psychology \& Marketing, 11, 4, 393-416.

Southerton D. (2001), Consuming kitchens: taste, context and identity formation, Journal of Consumer Culture, 1, 2, 179-203.

Stern P.C., Dietz T. et Kalof L. (1993), Value orientations, gender and environmental concern, Environment and Behavior, 25, 3, 322-348.

Stock J.R. (1992), Reverse logistics, Council of Logistics Management, Oak Brook, IL.

Taylor S. et Todd P. (1995), Understanding household garbage reduction behavior: a test of an integrated model, Journal of Public Policy and Marketing, 14, 2, 192-204.

Teller C., Kotzab H. et Grant D.B. (2012), The relevance of shopper logistics for consumers of store-based retail formats, Journal of Retailing and Consumer Services, 19, 1, 59-66.

Thøgersen J. (1997), Facilitating recycling: reverse-distribution channel design for participation and support, Social Marketing Quarterly, 4, 1, 42-55.

Tixier D., Mathe H. et Colin J. (1983), La logistique d'entreprise. Moyens, mécanismes et enjeux, Paris, Dunod.

Van de Ven A. H. (1992), Suggestions for studying strategy process: a research note, Strategic Management Journal, 13, 5, 169-188.

Vining J. et Ebreo A. (1990), What makes a recycler? A comparison of recyclers and nonrecyclers, Environment and Behavior, 22, 1, 55-73.

Weber M. (1965), Essais sur la théorie de la science, Plon, Paris.

Werner C.M. (1987), Home interiors: a time and place for interpersonal relationships, Environment and Behavior, 19, 169-179. 